\title{
Fissile Material Disposition Program Final Immobilization Form Assessment and Recommendation
}

\author{
S. G. Cochran \\ W. H. Dunlop \\ T. A. Edmunds \\ L. M. MacLean \\ T. H. Gould
}

October 3, 1997

This is an informal report intended primarily for internal or limited external distribution. The opinions and conclusions stated are those of the author and may or may not be those of the Laboratory.

Work performed under the auspices of the U.S. Department of Energy by the Lawrence Livermore National Laboratory under Contract W-7405-Eng-48. 


\section{DISCLAIMER}

This document was prepared as an account of work sponsored by an agency of the United States Government. Neither the United States Government nor the University of California nor any of their employees, makes any warranty, express or implied, or assumes any legal liability or responsibility for the accuracy, completeness, or usefulness of any information, apparatus, product, or process disclosed, or represents that its use would not infringe privately owned rights. Reference herein to any specific commercial product, process, or service by trade name, trademark, manufacturer, or otherwise, does not necessarily constitute or imply its endorsement, recommendation, or favoring by the United States Government or the University of California. The views and opinions of authors expressed herein do not necessarily state or reflect those of the United States Government or the University of California, and shall not be used for advertising or product endorsement purposes.

This report has been reproduced directly from the best available copy.

Available to DOE and DOE contractors from the Office of Scientific and Technical Information

P.O. Box 62, Oak Ridge, TN 37831

Prices available from (615) 576-8401, FTS 626-8401

Available to the public from the

National Technical Information Service

U.S. Department of Commerce 5285 Port Royal Rd.,

Springfield, VA 22161 


\title{
Fissile Material Disposition Program
}

Final Immobilization Form

\author{
Assessment \\ and \\ Recommendation
}

October 3, 1997

Stephen G. Cochran, LLNL

William H. Dunlop, LLNL

Thomas A. Edmunds, LLNL

Lee M. MacLean, LLNL

Thomas H. Gould, WSRC 



\section{TABLE OF CONTENTS}

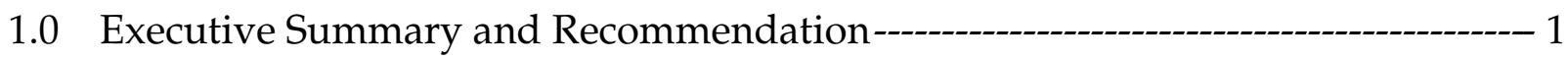

2.0 Introduction - 3

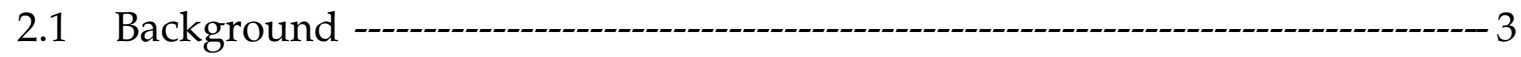

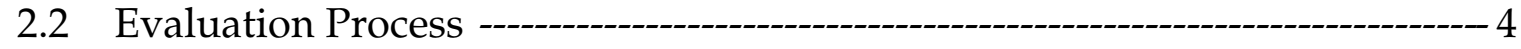

2.3 Documentation Supporting the Immobilization Technology Recommendation --- 5

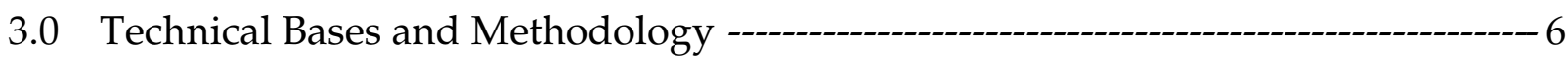

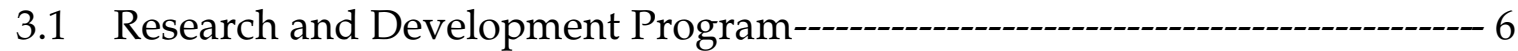

3.2 Decision Criteria -- 8

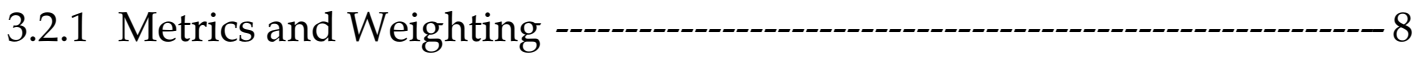

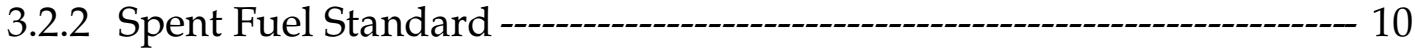

3.3 Technical Evaluation Panel Review ------------------------------------- 10

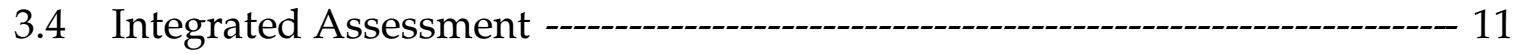

3.5 Peer Review --os 11

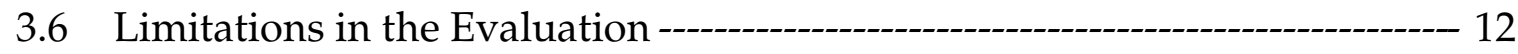

3.7 Bounding Conditions, Uncertainties and Assumptions-------_---_---_---- 12

3.7.1 ROD Related Assumptions ----on 12

3.7.2 Expected Plutonium Feed Materials and

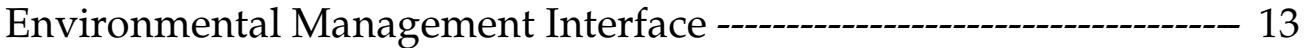

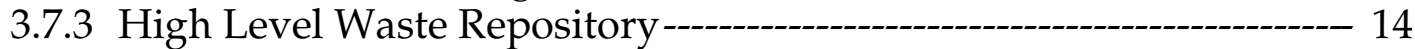

3.7.4 Schedule----o- 15

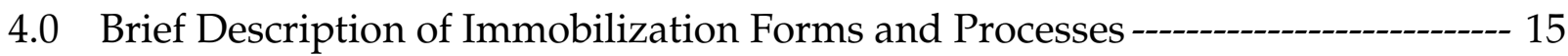

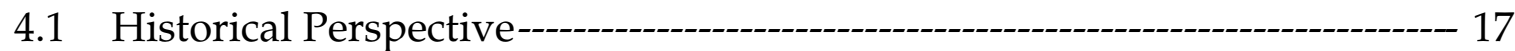

4.1.1 Glass---on 17

4.1.2 Ceramic--- 18

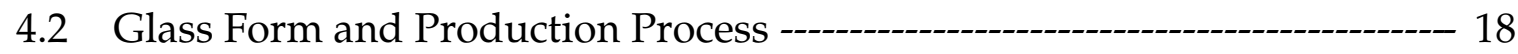

4.2.1 Baseline Glass Composition and Physical Properties------------------- 18

4.2.2 Baseline Glass Process --_ 20

4.3 Ceramic Form and Production Process--- 24

4.3.1 Baseline Ceramic Composition and Physical Properties ---------------- 24

4.3.2 Baseline Ceramic Process --_-_-_on 25

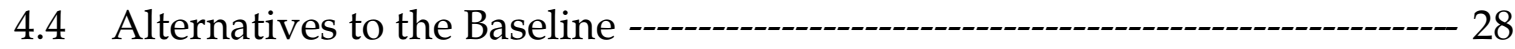

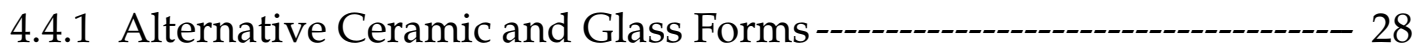

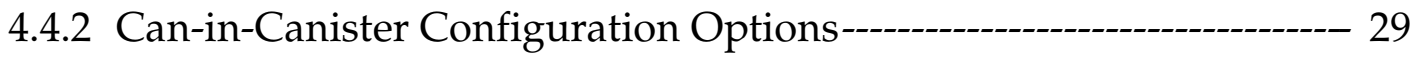

5.0 Assessment of Forms Against Criteria --_-_o 30

5.1 Ratings ------- 30

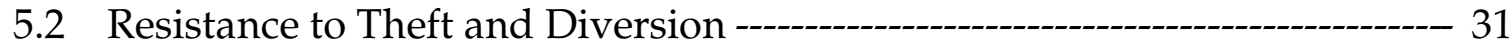

5.2.1 Low Inherent Attractiveness --_- 31

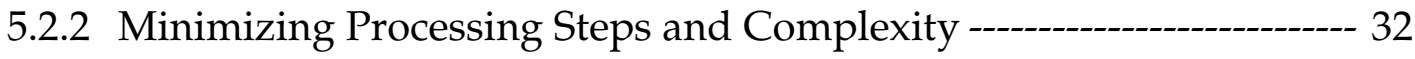

5.2.3 Safeguards and Security Assurance---_-_- 32 
5.2.4 Difficulty of Retrieval and Extraction by Rogue Party-------------- 32

5.3 Resistance to Retrieval, Extraction, and Reuse by the Host Nation --------- 35

5.4 Technical Viability: Repository Acceptability ----------------------------- 35

5.5 Environmental, Safety, and Health Compliance---------------------- 38

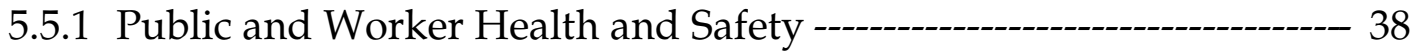

5.5.2 Waste Forms and Minimization---_-_-_-_- 41

5.6 Cost Effectiveness--- 41

5.6.1 Investment and Life Cycle Cost ---_-_- 42

5.6.2 Utilization of Existing Infrastructure----------------------------------- 45

5.7 Timeliness --- 46

5.7.1 Time to Start Disposition----_-

5.7.2 Time to Complete Disposition ---

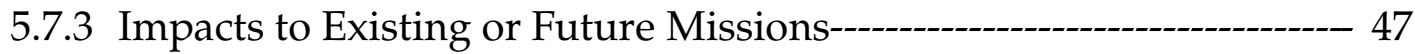

6.0 Overall Assessment --_-_- 48

6.1 Integrated Rating of Immobilization Forms Against Criteria----_------ 48

6.2 Robustness of Recommendation and Sensitivity Analysis ------------------- 49

6.2.1 Robustness to Programmatic and Technical Uncertainties ----------- 49

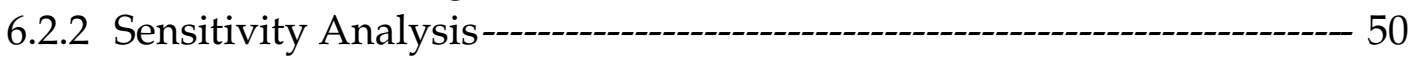

6.3 Peer Review Comments ---_-_-_-_-_-_- 51

6.4 Conclusion-- 52

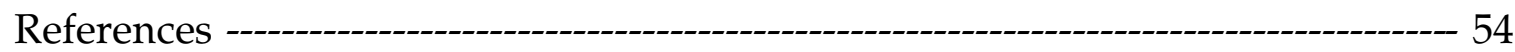

Appendix A Sensitivity Analysis --_- A-1 


\section{Final Immobilization Form Assessment and Recommendation}

\subsection{Executive Summary and Recommendation}

Lawrence Livermore National Laboratory (LLNL), in its role as the lead laboratory for the development of plutonium immobilization technologies for the Department of Energy's Office of Fissile Materials Disposition (MD), has been requested by $\mathrm{MD}$ to recommend the preferred immobilization technology for the disposition of excess weapons-usable plutonium. The recommendation and supporting documentation was requested to be provided by September 1, 1997 . This report addresses the choice between glass and ceramic technologies for immobilizing plutonium using the can-in-canister approach, providing a comparative evaluation of the two candidate technologies and a recommendation of the preferred form based on technical considerations.

The technologies for the candidate glass and ceramic forms were compared with respect to criteria and metrics that reflect programmatic and technical objectives through a series of three separate evaluations, each one building on the preceding evaluation(s):

- Evaluation of the R\&D and engineering data for the two forms with respect to the individual decision criteria/metrics by a technical evaluation panel (TEP) composed of experts from within the Immobilization Program.

- Integrated assessment by LLNL Immobilization Program management of the candidate technologies with respect to the weighted criteria and other programmatic objectives, leading to a recommendation to DOE/MD on the preferred technology based on technical factors (this report).

- Assessment of the decision process, evaluations, and recommendation by a peer review panel (PRP) of independent experts.

The criteria used to assess the relative merits of the two immobilization technologies are a subset of criteria previously used by MD to choose among several disposition options leading to the Programmatic Environmental Impact Statement and Record of Decision (ROD) for the Storage and Disposition of WeaponsUsable Fissile Materials, issued in January of 1997. The criteria are: (1) resistance to theft, diversion, and recovery of plutonium by a terrorist organization or rogue nation; (2) resistance to recovery and reuse by the host nation; (3) technical viability, including technical maturity, development risk, and acceptability of the form for repository disposal; (4) environmental, safety, and health factors; (5) cost effectiveness; and (6) timeliness. 
On the basis of the findings from the three technical evaluations, we recommend that the ceramic technology be developed for deployment in the planned plutonium immobilization plant using the can-in-canister approach. Both candidate technologies were found by the evaluators to be acceptable for the plutonium immobilization mission. However, the ceramic technology offers a number of important advantages over glass, notably:

- The ceramic form is more robust to the threat of theft, diversion, or reuse because the extraction of plutonium from the final form is more complicated and neither fully developed nor published.

- The ceramic form is expected to be more durable in a repository environment and should retain plutonium and its decay products for longer periods of time.

- The ceramic form has a significantly lower radiation source term that reduces the potential for worker exposure during fabrication before insertion into high-level waste canisters.

- The ceramic form and process offer potentially significant cost savings relative to the glass technology.

- The ceramic technology is more flexible, and can better accommodate modifications to programmatic and technical requirements.

The maturity of the ceramic technology, which was found to be comparable to that of glass, is sufficient to provide DOE with a reasonably high level of confidence that plutonium immobilization can be carried out successfully on the desired schedule. The principal technical issue for the glass technology is development of the high temperature induction-heated melter, and the principal technical issue for the ceramic is development of a product control strategy.

There are no "show stopper" issues for the ceramic technology (nor for the glass form). Therefore, we recommend that the research and development program be focused on the continued development of the ceramic immobilization technology for early deployment into the planned plutonium immobilization plant.

The results of the three evaluations are generally consistent. The PRP findings validate the results of the TEP assessment and those presented in this report, and endorse our recommendation for the deployment of the ceramic technology. 


\subsection{Introduction}

Lawrence Livermore National Laboratory (LLNL), in its role as the lead laboratory for the development of plutonium immobilization technologies for the Department of Energy's Office of Fissile Materials Disposition (MD), has been requested by $\mathrm{MD}$ to recommend an immobilization technology for the disposition of surplus weapons-usable plutonium. The recommendation and supporting documentation was requested to be provided by September 1, 1997. This report addresses the choice between glass and ceramic technologies for immobilizing plutonium using the can-in-canister approach. Its purpose is to provide a comparative evaluation of the two candidate technologies and to recommend a form based on technical considerations.

\subsection{Background}

As part of their agreement to make large reductions in nuclear weapons, the United States and Russia have undertaken joint programs to develop and implement approaches for ensuring the secure management, storage, and eventual disposition of the fissile materials rendered surplus by the arms reductions. These endeavors have been endorsed with the highest priority by the presidents of both countries. To demonstrate the U.S. government's commitment to these objectives, President Clinton declared in March, 1995 that approximately 50 metric tons (MT) of plutonium, including about 38 MT of weapon-grade material, was surplus to U.S. needs and would be set aside for eventual disposition. Russia has indicated that a similar quantity of plutonium will be made surplus.

In the U.S., the responsibility for fissile materials disposition resides with the DOE Office of Fissile Materials Disposition, which was created in 1994 to manage all DOE activities relating to the management, storage, and disposition of surplus fissile materials. For plutonium, the overall goal of the disposition program is to "make the plutonium as unattractive and inaccessible for retrieval and weapons use as the residual plutonium in the spent fuel from commercial reactors". This goal, referred to as the "Spent Fuel Standard," was originally stated in a report by the U.S. National Academy of Sciences (NAS) Committee on International Security and Arms Control (Ref. 1).

Since its inception, MD has been conducting development activities and evaluations of the most promising disposition technologies leading to a choice of the best one or two technologies for implementation. In the Record of Decision (ROD) for the Storage and Disposition of Weapons-Usable Fissile Materials , issued in January 1997 (Ref. 2), DOE announced its decision to pursue two alternative technologies for plutonium: (1) irradiation of plutonium as mixed-oxide fuel in existing power reactors, and (2) immobilization of plutonium into large solid forms containing fission products as a radiation barrier. The immobilization alternative will be used for the disposition of impure plutonium materials and 
could, if desired, be used for the larger quantity of pure plutonium materials from retired weapon components (pits).

During the course of assessing the numerous technology variants for immobilization as input to the PEIS/ROD process, six variants were chosen for further study (Refs. 3-9). In the ROD, DOE expressed preference for an immobilization approach that would "include the use of the can-in-canister option...for a portion of the surplus, non-pit plutonium material." In the can-incanister approach, cans of either glass or ceramic forms containing plutonium are encapsulated within canisters of high-level waste (HLW) glass, such as now produced in the Savannah River Site's (SRS) Defense Waste Processing Facility (DWPF). In a subsequent report (Ref. 10), LLNL concluded that the can-incanister (or external radiation barrier) approach with the plutonium immobilized in either glass or ceramic forms was technically superior to the other technology variants. As a consequence, the glass and ceramic technologies have been the focus of an aggressive research and development program aimed at selecting the final form by September 1997.

\subsection{Evaluation Process}

Evaluation of the candidate glass and ceramic technologies has involved the following steps:

- Development of experimental, engineering, and analytical data as part of ongoing immobilization research and development (R\&D) (October 1995July 1997).

- Definition of decision criteria and metrics, and their weights (relative importance), for comparing the glass and ceramic technologies (November 1996-January 1997, except for the weights).

- Evaluation of the R\&D data for the two forms with respect to the individual decision criteria/metrics by a technical evaluation panel (TEP) composed of experts from within the Immobilization Program (July 8-August 9, 1997).

- Integrated assessment by the lead laboratory (LLNL) of the candidate technologies with respect to the weighted criteria and other programmatic objectives, leading to a technology recommendation to $\mathrm{DOE} / \mathrm{MD}$ on the preferred technology (August 4-15, 1997).

- Assessment of the decision process, evaluations, and recommendation by a peer review panel (PRP) of independent experts (August 18-22, 1997).

The evaluation process is depicted in Figure 1; individual steps are described in Section 2.0. The overall evaluation, excluding the R\&D work and criteria development, was conducted over a seven-week period in July and August 1997. 
Figure 1. Immobilization form decision process.

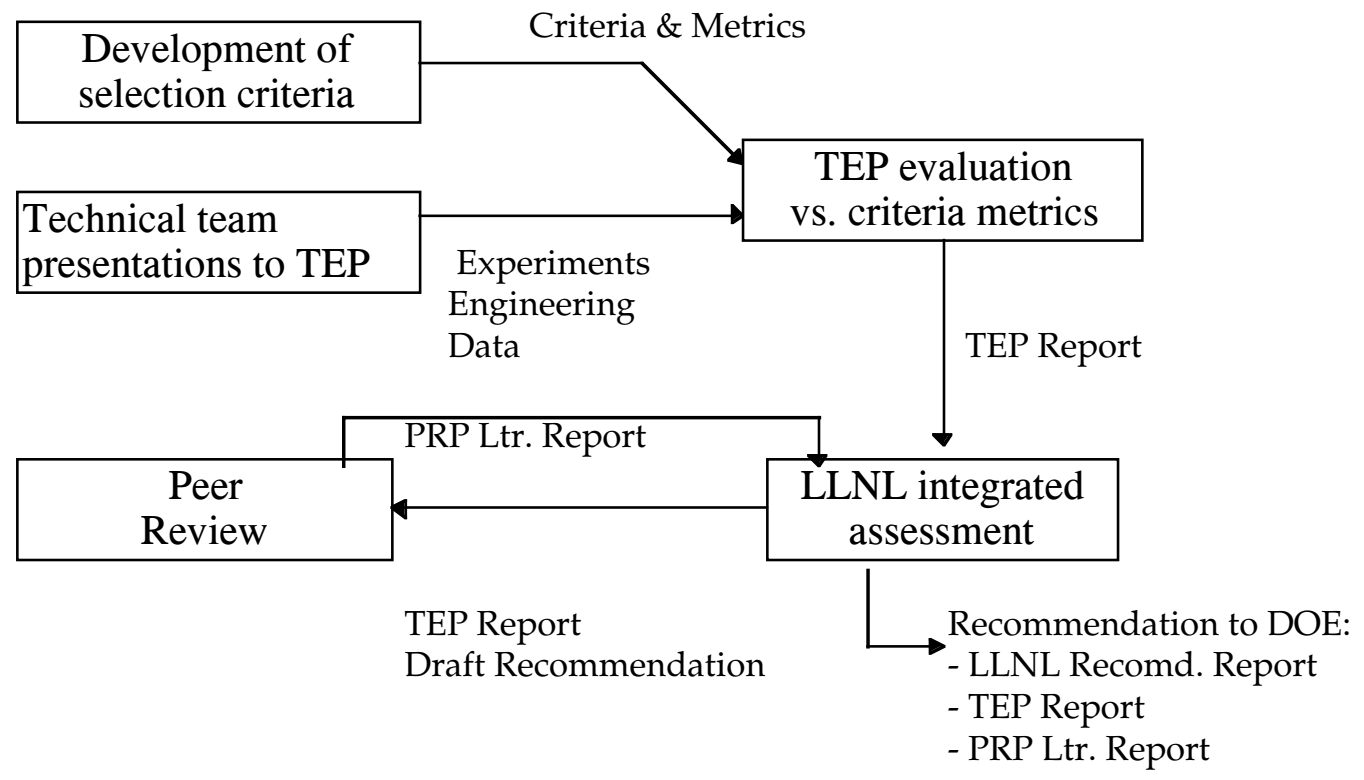

\subsection{Documentation Supporting the Immobilization Technology Recommendation}

Significant numbers of published and unpublished reports and presentation materials were used in evaluating the two immobilization technologies. This large body of information was distilled into the following reports, which provide the basis for our recommendation:

- Immobilization Technology Down-Selection: Radiation Barrier Approach, (May 23, 1997) (Ref. 10).

- Final Immobilization Form Assessment and Evaluation, (August 29, 1997) (this report).

- Technical Evaluation Panel Assessment of Glass and Ceramic Immobilization Technologies, (August 22, 1997) (Ref. 11).

- Letter Report of the Immobilization Technology Peer Review Panel, (August 22, 1997) (Ref. 12).

Key published and unpublished documents, analyses, and presentation materials used in the TEP and LLNL assessments have been organized into binders that will be retained by the LLNL Immobilization project office for future reference. 


\subsection{Technical Bases and Methodology}

\subsection{Research and Development Program}

The Immobilization R\&D Program was restructured in October 1996 to reflect the importance of expediting the implementation of technologies for the disposition of surplus weapons-usable plutonium in the U.S. This urgency was stressed in the DOE Office of Arms Control and Nonproliferation report on plutonium disposition alternatives (Ref. 13). The R\&D needed to choose between the candidate glass and ceramic immobilization technologies was rescoped and rescheduled to be completed by July 1997, some 14 months earlier than originally planned. The R\&D effort involved (Ref. 14):

- Glass and ceramic formulation development: developing the composition and fabrication parameters of the forms for the range of expected plutonium feed materials, and characterizing the important macrostructure and microstructure properties of the resulting experimental forms.

- Preliminary glass and ceramic process development: defining processing parameters, identifying equipment development needs, and performing early scale-up tests of the key fabrication steps for both forms.

- Characterization: evaluating the performance of the forms under accelerated and simulated repository conditions and developing chemical thermodynamic data for repository modeling studies.

- Preconceptual engineering: studies supporting the parallel Plutonium Disposition Environmental Impact Statement activity and providing a rough estimate of facility configuration and cost differences between the two technologies.

- Assessment: evaluating proliferation resistance of the can-in-canister waste form for both glass and ceramic forms vis-a-vis spent nuclear fuel.

These experimental, analytical, and engineering studies provided a broad set of data on the two forms and their processes that were used to evaluate the two technologies against the criteria and metrics described in section 3.2.

For the longer term, the program participants have jointly prepared an integrated immobilization R\&D plan (Ref. 15) that couples the R\&D activities to the overall project schedule for immobilization (Figure 2). The R\&D plan defines detailed tasks for the development of the form, process parameters, and equipment needed to support the design of the immobilization plant. The plan also identifies and schedules the waste form qualification activities that will be required to obtain acceptance of the can-in-canister form by the Civilian Radioactive Waste Management System. 
Five laboratory organizations are involved in this $R \& D$ program with the following responsibilities (some are shared):

- Lawrence Livermore National Laboratory (LLNL): overall technical lead for the R\&D program, ceramic form development lead, glass form development support, process/equipment development with plutonium, and form characterization and qualification.

- Westinghouse Savannah River Company's (WSRC) Technology Center: glass form development lead, ceramic formulation support, preferred immobilization site, and process/equipment development.

- Argonne National Laboratory (ANL): form characterization and glass formulation support.

- Australian National Science and Technology Organization (ANSTO): support of LLNL on ceramic form development.

- Pacific Northwest National Laboratory (PNNL): form characterization and glass formulation support.

Figure 2. Immobilization project schedule.

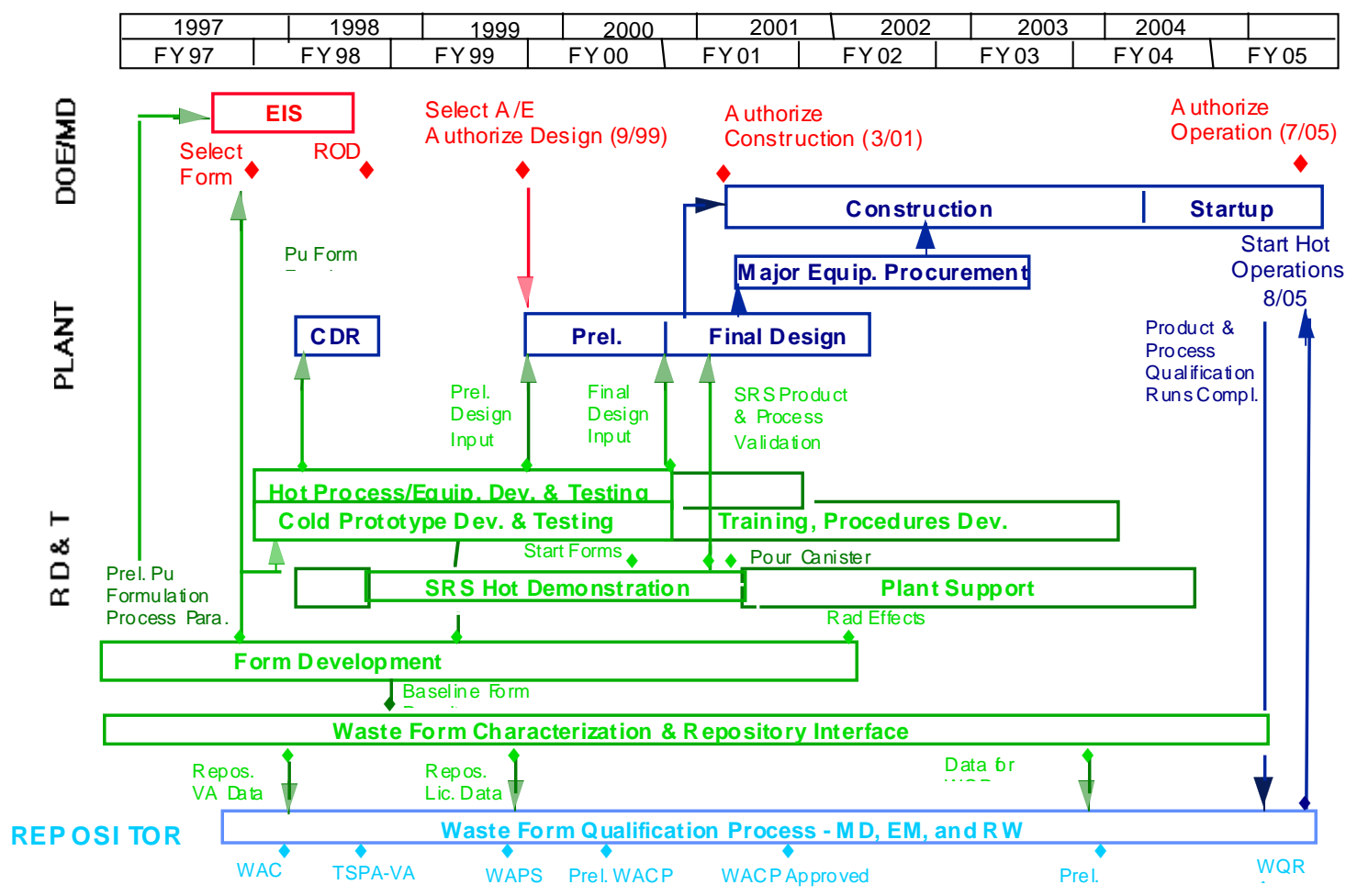




\subsection{Decision Criteria}

The criteria used to evaluate the immobilization technology variants are a subset of those that have been used by the Fissile Materials Disposition Program in previous studies and decisions (Ref. 16), namely:

- Resistance to theft and diversion by unauthorized parties. Each step in the disposition process must be capable of providing comprehensive protection and control of weapons-usable fissile materials.

- Resistance to retrieval, extraction, and reuse by the host nation. The surplus material must be made highly resistant to potential use in weapons to reduce reliance on institutional controls and to demonstrate that the arms reduction will not be easily reversed.

- Technical viability. There should be a high degree of confidence that the alternative will be technically successful.

- Environment, safety, and health (ESEH). High standards of public and worker safety and environmental protection must be met, and significant additional ES\&H burdens should not be created.

- Cost effectiveness. Disposition should be accomplished in a cost-effective manner and be compatible with reasonable long-term storage alternatives.

- Timeliness. There is an urgent need to begin plutonium disposition and minimize the time period the surplus fissile materials remain in weaponsusable forms.

Three nontechnical criteria used by DOE in previous alternative assessments (fostering cooperation with Russia, public and institutional acceptance, and additional benefits) were not considered in the evaluations by LLNL and the TEP. Our intent was to provide DOE with a recommendation based solely on the technical merits of the two technologies. Political and institutional considerations in selecting the final immobilization form rest with the ultimate decision maker, DOE.

\subsubsection{Metrics and Weighting}

Factors and metrics for each criterion were developed by a committee of experts from the Immobilization Development Team representing LLNL, ANL, PNNL, SRS, and the Office of Civilian Radioactive Waste (RW) M\&O contractor. The criteria and major factors are summarized in Table 1; they are described fully in References 10 and 11. MD provided criteria weights reflecting the relative level of importance of the various criteria (Ref. 17); they are shown in parentheses in Table 1. 
Table 1. Decision criteria and factors.

Criterion 1. Resistance to theft or diversion by unauthorized parties (0.22)

a. Low inherent attractiveness

b. Minimization of transportation, facilities, and sites

c. Minimization of processing

d. Safeguards and security assurance

e. Difficulty of retrieval, extraction, and use by a clandestine group or rogue nation

Criterion 2. Resistance to retrieval, extraction, and reuse by the host nation (0.19)

a. Difficulty of retrieval, extraction, and reuse

b. Assurance of detection of diversion and extraction

Criterion 3. Technical viability (0.19)

a. Technical maturity (considered as impacts on cost and timeliness)

b. Viability risks (considered as impacts on cost and timeliness)

c. Repository acceptability of disposal form

Criterion 4. Environmental, safety, and health compliance (0.14)
a. Public and worker health and safety
b. Waste minimization
d. Known and manageable waste forms

Criterion 5. Cost effectiveness (0.11)
a. Life cycle cost:
b. Investment and start-up cost
c. Establish product acceptability requirements
d. Potential for cost sharing. dollars
e. Utilization of existing Infrastructure
f. Cost estimate certainty

Criterion 6. Timeliness $(0.15)$
a. Time to start disposition/time to open facility
b. Time to complete
c. Impacts on existing or future missions

Several of the factors are interrelated. For example, the technical viability factors of maturity and risk could be expressed in terms of impacts and risks to schedule (timeliness) and to cost. At the request of MD (Ref. 17), the cost and schedule impacts of associated with technical maturity are considered in the subsequent sections where the two form technologies are assessed against the criteria/factors. Similarly, the ES\&H factor of radiation exposure, which is a potential discriminator, has cost impacts (shielding, automation, boron enrichment, and space to reduce dose). 


\subsubsection{Spent Fuel Standard}

Criteria 1 and 2 are related to the higher level NAS Spent Fuel Standard (Ref. 1) adopted by MD. That is, the can-in-canister immobilization form must "make the plutonium as unattractive and inaccessible for retrieval and weapons use as the residual plutonium in the spent fuel from commercial reactors." The attributes of the Spent Fuel Standard include:

- High radiation dose rate: use of fission products from reactor fuel to achieve doses $>100 \mathrm{rem} /$ hour one meter from the canister surface 30 years after fabrication.

- Large and heavy integral assembly: impossible for an individual to move without heavy equipment.

- Dilute distribution of plutonium-bearing materials within the solid matrix containing fission products.

- Higher concentration of heavier plutonium isotopes than weapon-grade plutonium.

\subsection{Technical Evaluation Panel Review}

The initial step in evaluating the relative merits of the glass and ceramic immobilization technologies was a comprehensive review of the technologies during a two-week period in late July, 1997. A panel of experts from the five participating laboratories involved in the immobilization project performed the review. The Technical Evaluation Panel (TEP) reviewed the performance of the glass and ceramic form technologies relative to the stated criteria and metrics. The eight panel members represented the key technology areas associated with the two technologies:

- Booth Myers (LLNL): chairman (plutonium facilities).

- Carol Jantzen (WSRC) and Adam Jostsons (ANSTO): glass and ceramic (mineral) science and formulation.

- Mal McKibben (WSRC) and Guy Armantrout (LLNL): glass and ceramic processing.

- Denis Strachan (ANL), John Vienn (PNNL), and Henry Shaw (LLNL): form characterization and repository performance.

The TEP received data in oral briefings and in written format during the week of July 28. During the following week, the members evaluated the attributes of the glass and ceramic technologies for each of the criteria/metrics, based on the information provided, and then documented their findings in a detailed report (Ref. 11). Section 5.0 discusses the TEP findings. 


\subsection{Integrated Assessment}

Information presented to the TEP and the TEP report provided the basis for the LLNL integrated assessment described in this report. In this integrated assessment, performed during the period July 28-August 15, 1997 the cumulative merits of the two technologies were evaluated against the weighted decision criteria. Additionally, a sensitivity analysis of results was performed by converting the qualitative ratings of the technologies to a numerical scale. This integrated assessment is discussed in Section 6.0.

\subsection{Peer Review}

A panel of five independent experts was assembled to review the decision process, data, analyses, and conclusions presented in the TEP report and this report during the week of August 18, 1997. In addition to reviewing these two reports, the Peer Review Panel (PRP) received information in the form of briefings by LLNL and WSRC and briefings by and discussions with the TEP members. The panel's findings are summarized and addressed in Section 6.4 and are presented in a letter report (Ref. 12).

The PRP members, listed below, were chosen based on their expertise in the key technology areas important to the decision:

- Mr. Matthew Bunn, Harvard Kennedy School of Government, chairman: nonproliferation.

- Dr. Donald Langmuir, consultant and adjunct professor, Colorado School of Mines: geochemistry and waste form corrosion.

- Dr. Ronald Loehman, head of University of New Mexico/Sandia National Laboratory Advanced Materials Laboratory: ceramics and glass science and technology.

- Dr. David Stahl, Manager, Yucca Mountain Project Waste Package Materials Dept., Framatome/B\&W: repository performance.

- Mr. Alan Williams, Cconsultant, Bechtel National: plutonium processing and facilities. 


\subsection{Limitations in the Evaluations}

Three major constraints that placed limitations on the evaluations that were performed to arrive at the recommendation documented by this report. They are related to the availability of data, the specificity and clarity of the criteria and metrics, and the severe time limits placed on the evaluation process.

Because the immobilization form decision was accelerated by one year, some of the experiments and engineering studies originally planned could not be completed in time for this recommendation. For example, cost estimates for the baseline glass and ceramic immobilization facilities will not be completed until FY 1998. Nonetheless, there was sufficient information for the reviewers to make reasonable comparisons for most of the decision factors. A number of the decision factors were qualitative or not sufficiently specific. Comparative evaluations of the two forms against such factors required significant interpretation and technical judgment. Finally, the entire evaluation process, excluding organizational activities, was limited to a four-week period of intensive activity. In spite of these limitations, there were adequate data and sufficient differences between the two technologies to arrive at a meaningful recommendation.

\subsection{Bounding conditions, uncertainties, and assumptions.}

Because of programmatic uncertainties, a number of important assumptions had to be made for the purposes of the TEP review and this overall assessment. They are related to the preferred immobilization implementation paths expressed in the ROD (Ref. 2), the MD program interfaces with the DOE Environmental Management (EM) and Civilian Radioactive Waste Management (RW) programs, and the planned project schedule for implementing the plutonium immobilization alternative.

\subsubsection{ROD-Related Assumptions}

1. The PEIS-ROD and May 1997 Notice of Intent (NOI) for the Plutonium Disposition Environmental Impact Statement (Ref. 18) have identified the can-in-canister variants (glass and ceramic) as the preferred immobilization technology. In the can-in-canister approach (Figure 3), cans of either glass or ceramic forms containing plutonium are encapsulated within canisters of high-level waste (HLW) glass, such as now produced in the Savannah River Site (SRS) Defense Waste Processing Facility (DWPF). The glass and ceramic forms that incorporate the plutonium materials are specially formulated materials designed

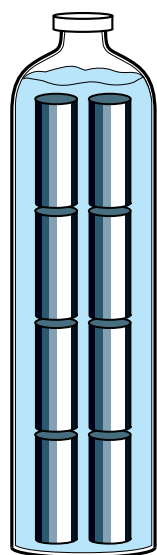

Figure 3. Schematic Diagram of the can-in canister configuration. 
to accommodate large quantities of actinides and achieve high levels of durability. During the past year, the Immobilization Program has focused on the development of these can-in-canister based forms.

2. The May 1997 NOI (Ref. 18) identified the SRS as the preferred site for the immobilization mission, primarily because of the existence of the operating DWPF that is vitrifying HLW. For this exercise, the evaluators assumed that the plutonium (first stage) immobilization plant will be located at SRS and that standard DWPF canisters will be used as the vessels for the cans of plutonium forms and HLW glass. It was further assumed that the Immobilization Program would have minimal impacts on the overall DWPF mission. Characteristics of the HLW glass canister are given in Table 2.

Table 2. Physical characteristics of DWPF HLW-glass canister.

\begin{tabular}{ll}
\hline Parameter & Value \\
\hline Canister outer diameter & $0.6 \mathrm{~m}(24 \mathrm{in})$. \\
Canister inner diameter & $0.591 \mathrm{~m}(23.25 \mathrm{in})$. \\
Overall canister height & $3.0 \mathrm{~m}(10 \mathrm{ft})$ \\
Nominal pour height of HLW glass & $2.3-2.4 \mathrm{~m}(90-96 \mathrm{in} . \mathrm{s})$ \\
Mass of HLW glass in full canister & $\sim 1680 \mathrm{~kg}$ \\
Density of HLW glass & $\sim 2.7 \mathrm{~g} / \mathrm{cm}^{3}$ \\
\hline
\end{tabular}

\subsubsection{Expected Plutonium Feed Materials and Environmental Management Interface}

For this and the TEP assessments, two plutonium feed cases for the planned immobilization plant were considered based on the possible partitioning of the surplus plutonium between the immobilization and reactor-MOX alternatives:

- 50-MT feed where all the surplus plutonium materials come to the immobilization plant (baseline feed case 1).

- 18.2 MT feed, commonly referred to as the "17-MT case," where only nonweapons-pit materials come to the immobilization plant (baseline feed case 2).

The non-pit materials include impure metals, alloys, and predominately oxide materials that are currently within the Environmental Management Stabilization Program, as well as pure metal and oxides still under the control of Defense Programs. Other plutonium residues within the Environmental Management Stabilization Program may also be candidates for eventual immobilization. In general, it is assumed that the Environmental Management materials will be in stabilized forms that contain greater than 50 weight percent plutonium. Table 3 summarizes the categories and quantities of expected feed materials; additional details on the impurity contents are given in Reference 19 and in the TEP report (Table 3.2.2). 
Table 3. General categories and quantities of surplus plutonium (Ref. 20).

\begin{tabular}{lccc}
\hline Category & MT Pu & MT U & MT Other \\
\hline Weapons Pits Oxide & 31.8 & 0 & 0 \\
Metals & 3.4 & 0 & 0.1 \\
Non-pit Oxides & 9.0 & 2.0 & 1.2 \\
Alloys & 1.0 & 0.3 & 0.1 \\
Fuels (unirradiated) & 4.8 & 14.5 & 0.1 \\
\hline Total & 50 & 17 & 1.5 \\
\hline
\end{tabular}

The plutonium feed materials will be grossly blended to homogenize the 17 MT of uranium from the unirradiated fuel materials. This level of blending will also degrade the plutonium isotopics by mixing weapon-grade with fuel-grade plutonium. Additionally, some limited blending may be performed, if needed, to reduce the concentrations of any impurities that are difficult to immobilize. The non-uranium impurities in the plutonium feed represent about $2.6 \%$ and $4.5 \%$ by weight of the bulk feed for the $50 \mathrm{MT}$ and $17 \mathrm{MT}$ cases, respectively.

\subsubsection{High Level Waste Repository}

DOE intends that the can-in-canister immobilization forms will be combined with the normal DWPF-generated HLW-glass canisters for eventual disposal in the Yucca Mountain repository. The final design of the waste package for the HLW canisters, including the ones containing immobilized plutonium, has not been established, nor has the arrangement of the HLW packages relative to the spent fuel packages in the repository. The currently preferred concept involves corrosion-resistant waste packages containing four to five HLW canisters that would be emplaced horizontally in the repository drifts.

Another uncertainty involves the repository acceptance requirements for the immobilization form, which have not been established. Acceptance criteria for the DWPF glass form have been established. To some extent, they were based on the requirement that the waste package and repository system retain the HLW's fission products for several half lives, or several hundred years. The can-in-canister immobilization form will contain significantly larger quantities of fissile elements (primarily $\mathrm{Pu}-239$ which decays with a half-life of $\sim 24,000$ years to the fissile isotope U235 which has a half- life of $\sim 700$ million years). Therefore, there is an added concern for the immobilization form regarding the possibility of criticality events occurring in the repository system during a period of millions of years. Migration of immobilization radionuclides to the biosphere, as pointed out in the TEP report (Ref. 11), appears not to be an issue.

As a consequence of the criticality issue, it is possible that more stringent durability requirements could be placed on the plutonium form than exist for the HLW-glass 
form. At a minimum, the immobilized form will be required to comply with the existing HLW standards. As such, it is important to provide stringent control over the fabrication process to ensure a reliable product of sufficient quality.

\subsubsection{Schedule}

The goal of the Immobilization Program is to begin fabricating immobilized forms in the year 2005. To meet this objective, facility design will need to begin in FY 2000. The current R\&D plan (Section 3.1; Ref. 15) lays out development activities for both candidate forms that will meet this aggressive schedule (Figure 2). Significant delays in development of the form/processing technology or in the characterization testing and qualification process for repository acceptance would, in turn, delay the startup of the immobilization plant.

\subsection{Brief Descriptions of Immobilization Forms and Processes}

This section summarizes the basic attributes of the glass and ceramic candidate forms, their production processes, and their can-in-canister configurations. A more detailed description is presented in the TEP report (Ref. 11). The proposed glass form is a single-phase (in the baseline formulation) lanthanide borosilicate (LaBS) glass specially formulated to accommodate high concentrations of actinide elements $(\sim 16 \mathrm{wt} \%)$. The proposed ceramic form is a multiple phase titanate-based crystalline ceramic material synthesized to simulate durable titanate minerals existing in nature. These mineral-like titanate phases can accommodate actinide elements as high as 30 to 50 weight percent within their crystalline structures. Both forms are quite robust with respect to nonactinide impurities. Table 4 compares key attributes of the two forms for both the 50MT and 17-MT plutonium feed cases. Table 5 compares processing steps for the two forms. 
Table 4. Baseline feed streams and process operations for immobilization of Pu in ceramic or glass.

50 MT Pu with 16 MT U

17 MT Pu with 16 MT U

\begin{tabular}{|c|c|c|c|c|}
\hline Parameter & Ceramic & Glass & Ceramic & Glass \\
\hline Processing Approach & press/sinter & melt/stir & press/sinter & melt/stir \\
\hline $\begin{array}{l}\text { Nominal } \mathrm{Pu} \mathrm{O}_{2} \text { Feed Particle } \\
\text { Size }(\mu \mathrm{m})\end{array}$ & 20 & 20 & 20 & 20 \\
\hline Processing Temp. $\left({ }^{\circ} \mathrm{C}\right)$ & $1350+50 /-25$ & $1500 \pm 50$ & $1350+50 /-25$ & $1500 \pm 50$ \\
\hline Product Form & $\begin{array}{l}\text { sintered ceramic } \\
>92 \% \text { theoretical density }\end{array}$ & LaBS glass & $\begin{array}{l}\text { sintered ceramic } \\
>92 \% \text { theoretical density }\end{array}$ & LaBS glass \\
\hline $\begin{array}{l}\text { Product Form Density } \\
\left(\mathrm{gm} / \mathrm{cm}^{3}\right)\end{array}$ & 5.5 & 3.8 & 5.5 & 3.8 \\
\hline Product Phases & mixed mineral $^{\mathrm{a}}$ & homogeneous & mixed mineral $^{\mathrm{a}}$ & homogeneous \\
\hline $\begin{array}{l}\text { Pu Loading } \\
\text { (wt\% elemental) }\end{array}$ & 10.5 & 8.8 & 10.5 & 7.1 \\
\hline $\begin{array}{l}\text { Actinide Loading Tolerance } \\
(\mathrm{Pu}+\mathrm{U} \mathrm{wt} \% \text { elemental })\end{array}$ & 31.4 & 13.0 & 31.4 & 13.8 \\
\hline $\begin{array}{l}\text { Planned }{ }^{238} \mathrm{U} \text { addition } \\
\text { (wt\% elemental) }\end{array}$ & 17.6 & 0 & 11.0 & 0 \\
\hline $\begin{array}{l}\text { Tested non-actinide Impurity } \\
\text { Tolerance (wt } \% \text { oxide) }\end{array}$ & 13 & 6 & 13 & 6 \\
\hline $\begin{array}{l}\text { Outer Product Can } \\
\text { Dimensions } \\
\text { (diameter/height) }\end{array}$ & $3 " / 21 "$ & $4.5 " / 21 "$ & $3 " / 21 "$ & $4.5 " / 21 "$ \\
\hline $\begin{array}{l}\text { Numbers of Cans per } \\
\text { Canister }\end{array}$ & 28 & 16 & 28 & 16 \\
\hline $\mathrm{Pu}$ (Kg total) per Canister & 29 & 21 & 29 & 15 \\
\hline
\end{tabular}

a. Mineral phases identified in order of descending concentration include Pyrochlore, Zirconolite, Brannerite, Rutile, Silicate, and (Pu, Ti, Hf, Zr) mixed oxide phases. 
Table 5. Comparison of baseline ceramic and LaBS glass processes.

\begin{tabular}{|c|c|}
\hline Ceramic & LaBS Glass \\
\hline $\begin{array}{c}\text { Conditioning mill to size } \\
\text { reduce } \mathrm{UO}_{2} \text { and } \mathrm{PuO}_{2}\end{array}$ & \\
\hline $\begin{array}{c}\mathrm{Attritor} \text { mill to blend } \\
\mathrm{PuO}_{2} / \mathrm{UO}_{2} \text { and Oxide } \\
\text { precursor }\end{array}$ & $\begin{array}{c}\text { Attritor mill to co-grind } \\
\mathrm{PuO}_{2} / \mathrm{UO}_{2} \text { and glass } \\
\text { making frit }\end{array}$ \\
\hline Granulator & $\begin{array}{c}\text { Screw feeder to seven } \\
\text { melters }\end{array}$ \\
\hline Feed hopper to single press & Melter feed hopper \\
\hline Cold press & $\begin{array}{c}\text { Melters for vitrification } \\
\text { including off-gas system }\end{array}$ \\
\hline $\begin{array}{c}\text { Conveyer to six furnaces } \\
\text { into cans }\end{array}$ & Glass pour \\
\hline $\begin{array}{c}\text { Sintering furnaces including } \\
\text { off-gas system and } \mathrm{Ar} \\
\text { purge }\end{array}$ & $\begin{array}{c}\text { Can cool down } \\
\text { Disc cool down }\end{array}$ \\
\hline Inspection & Trim can \\
\hline Load discs into can & Load glass can in outer can \\
\hline Bagless loadout & Bagless loadout \\
\hline
\end{tabular}

\subsection{Historical Perspective}

\subsubsection{Glass}

Borosilicate glasses have been extensively used to immobilize high level and low level wastes in the U.S. and Europe (see Ref. 11 for citations to numerous publications). These glasses were chosen for their high flexibility to accommodate a broad range of chemicals, both liquids and solids, combined with acceptable durability for retaining fission products over their lifetimes under expected repository conditions. The borosilicate glasses developed for these waste disposal missions do not contain significant concentrations of actinide elements, because these materials are separated from the HLW during spent fuel processing. Generally, these borosilicate glasses are melted and cast at $1050^{\circ}$ to $1150^{\circ} \mathrm{C}$.

The LaBS glass was developed by WSRC originally to provide a storage form for americium and curium, and subsequently to provide an immobilization form for surplus plutonium. The objective was to find a glass composition that could accommodate large actinide concentrations, as well as the impurity elements in the surplus plutonium materials, and achieve acceptable durability. This was accomplished through the modification of the lanthanide containing Löffler glass (Löffler, 1932, U.S. Patent 2150694). As shown in the TEP report, the LaBS glass is able to accept combined actinide ( $\mathrm{Pu}$ and $\mathrm{U}$ ) concentrations of up to 16 weight percent 
along with the expected range of impurity elements. Leaching tests indicate that the baseline LaBS glass has higher durability than both the EA Standard Glass and the DWPF HLW glass. Because of the high lanthanide and actinide content in the LaBS glass, the melting temperature is much higher than that for the traditional borosilicate waste glasses, $1500^{\circ}-1550^{\circ} \mathrm{C}$. Characteristics of this new plutonium-containing glass form and fabrication process are briefly described in Section 4.2.

\subsubsection{Ceramic}

The proposed titanate-based crystalline ceramic form originated from the SYNROC (synthetic rock) forms developed and tested by the Australian National Science and Technology Organization (ANSTO) for a wide variety of wastes, such as the PW- $4 \mathrm{~b}$ wastes (SYNROC C), the SRS defense wastes (SYNROC D), and actinide-containing waste (SYNROC F). (Ref. 11 cites several references for these forms). These forms are much more durable than glasses under simulated repository conditions, but lack the flexibility of borosilicate glass to accommodate the broad range of chemical constituents contained in many waste streams. Furthermore, the hot pressing fabrication technology for these forms is more complex than the glass forming operations for shielded cell operations with HLW (Ref. 20).

With the advent of the plutonium immobilization mission, the SYNROC concept of incorporating radioactive elements into titanate-based mineral phases was adapted to plutonium. Both ANSTO and LLNL explored zirconolite-rich and pyrochlore/zirconolite titanate ceramics for incorporating plutonium and uranium. These phases proved to be extremely flexible for accepting high concentrations of the actinide elements as well as the expected range of impurities in the surplus plutonium feed materials. They also proved to be even more durable than the SYNROC C and D forms. LLNL chose to pursue a pyrochlore-rich ceramic that also contained zirconolite, brannerite, and rutile as secondary and tertiary phases. A cold press and sintering process, which is very similar to the MOX fuel processes in use in Europe, was developed for both the zirconolite- and pyrochlore-based forms. Thus, for the plutonium immobilization mission, the ceramic technology offers a compositionflexible form with extremely high durability, fabricated by a process that is no more complex than the LaBS glass process. The baseline composition, properties, and fabrication process of the ceramic form are briefly described in Section 4.3.

\subsection{Glass Form and Production Process}

\subsubsection{Baseline Glass Composition and Physical Properties}

For the glass baseline, a homogeneous single phase lanthanide borosilicate (LaBS) glass was developed for the vitrification of surplus fissile materials. Ingredients in the baseline LaBS glass are $\mathrm{Al}_{2} \mathrm{O}_{3^{\prime}}, \mathrm{B}_{2} \mathrm{O}_{3}, \mathrm{Gd}_{2} \mathrm{O}_{3}, \mathrm{HfO}_{2}, \mathrm{La}_{2} \mathrm{O}_{3}, \mathrm{Nd}_{2} \mathrm{O}_{3}$, $\mathrm{SiO}_{2}$, and $\mathrm{SrO}$, in addition to $\mathrm{PuO}_{2}$ and $\mathrm{UO}_{3}$. The hafnium and gadolinium are effective neutron absorbers for the repository environment. 
There are two baseline cases to account for the two feed variants. Baseline Case 1 assumes $50 \mathrm{MT}$ of plutonium feed and Baseline Case 2 assumes $17 \mathrm{MT}$ of plutonium feed. The two baseline cases differ only in the relative amount of impurities in the feed streams. The frit has been shown to be extremely tolerant to feed variations, thereby minimizing plutonium feed blending concerns based on impurity tolerances. The frit is also flexible to variations in the $\mathrm{UO}_{3}$ concentration of the incoming baseline feeds (Ref. 11).

When the LaBS baseline frit is mixed with the two different baseline feed compositions at different plutonium and uranium loadings, it produces glasses of slightly different composition. The final glass compositions for LaBS glass for each baseline case are shown in Table 6. For the 50 MT case, the baseline LaBS glass feed loadings consist of $10 \mathrm{wt} \% \mathrm{PuO}_{2}$ (8.8 $\mathrm{wt} \%$ plutonium), $3 \mathrm{wt} \% \mathrm{UO}_{3}(2.5$ wt $\%$ uranium), and up to $3 \mathrm{wt} \%$ impurities on an oxide basis. The 50 MT LaBS composition is, therefore, designated as $10 / 3 / 3$ and has the following neutron absorber to plutonium molar ratios: hafnium:plutonium $=0.71: 1.0$, gadolinium:plutonium=1.61:1.0, and boron:plutonium=8.2:1.0.

For the $17 \mathrm{MT}$ case, the baseline LaBS glass feed loadings consist of $8 \mathrm{wt} \% \mathrm{PuO}_{2}$ (7.1 wt\% plutonium), $8 \mathrm{wt} \% \mathrm{UO}_{3}(6.7 \mathrm{wt} \% \mathrm{U}$ ), and up to $3 \mathrm{wt} \%$ impurities on an oxide basis. The 17 MT LaBS composition is, therefore, designated as 8/8/3 and has the following neutron absorber to plutonium molar ratios: hafnium:plutonium=0.85:1.0, gadolinium:plutonium=1.94:1.0, and boron:plutonium=8.0:1.

In the glass immobilization process, $\mathrm{PuO}_{2}$ and $\mathrm{UO}_{x}$ are mixed with a prefabricated, prefused baseline frit containing the neutron absorbers hafnium and gadolinium. The co-ground frit and $\mathrm{PuO}_{2} / \mathrm{UO}_{\mathrm{x}}$ feed are fed directly to a melter and melted at $1500^{\circ} \mathrm{C}$, poured into cans, and allowed to cool. The LaBS glass has a measured density of $3.85 \mathrm{~g} / \mathrm{cm}^{3}$. The glass contains no open porosity but may exhibit some thermal stresses on cooling unless it is annealed. Unannealed glass can exhibit some cracking, (as does the HLW glass) with minimal effect on the glass durability. 
Table 6. Comparison of the final glass compositions for LaBS glass baseline for $50 \mathrm{MT}$ and $17 \mathrm{MT}$ plutonium immobilization.

\begin{tabular}{|c|c|c|}
\hline Oxide & LaBS 50MT baseline & LaBS 17MT baseline \\
\hline & & \\
\hline $\mathrm{Al}_{2} \mathrm{O}_{3}$ & 17.9 & 17.3 \\
\hline $\mathrm{B}_{2} \mathrm{O}_{3}$ & 9.7 & 9.4 \\
\hline $\mathrm{Gd}_{2} \mathrm{O}_{3}$ & 10.8 & 10.4 \\
\hline $\mathrm{HfO}_{2}$ & 5.5 & 5.3 \\
\hline $\mathrm{PuO}_{2}$ & 10.0 & 8 \\
\hline $\mathrm{La}_{2} \mathrm{O}_{3}$ & 6.8 & 6.6 \\
\hline $\mathrm{Nd}_{2} \mathrm{O}_{3}$ & 6.9 & 6.6 \\
\hline $\mathrm{SiO}_{2}$ & 24.3 & 23.4 \\
\hline $\mathrm{SrO}$ & 2.1 & 2.0 \\
\hline $\mathrm{UO}$ & 3 & 8 \\
\hline $\mathrm{Impurities}$ & 3 & 3 \\
\hline & & \\
\hline Total & 100.0 & 100.0 \\
\hline & & \\
\hline
\end{tabular}

\subsubsection{Baseline Glass Process}

The baseline LaBS glass process (less the head end plutonium feed conversion and preparation operations common to both glass and ceramic) is depicted schematically in Figure 4. The production process will operate in a batch mode to facilitate material accountability and to avoid criticality. The non-radioactive plant feeds are stored outside the glass processing facility.

Essentially all material handling must be performed with automated, hands-off, equipment in heavily shielded glove boxes. Glove-box systems similar to those in MOX fuel fabrication facilities are required. As will be discussed in Section 5.2, the $(\alpha, n)$ reaction in boron, a major constituent in the LaBS glass, produces a high neutron radiation field which requires special design considerations for the layout of equipment. With automated handling and 7-inch shielded glove boxes, the operators should receive acceptably low hand and whole body exposures during normal operation (primarily because most of the operator's time will not be spent near the glovebox line). For non-routine maintenance, exposures can be kept acceptably low by a combination of design (e.g., added space between glove boxes), temporary shielding, and administrative controls. 
Figure 4. Baseline glass process.

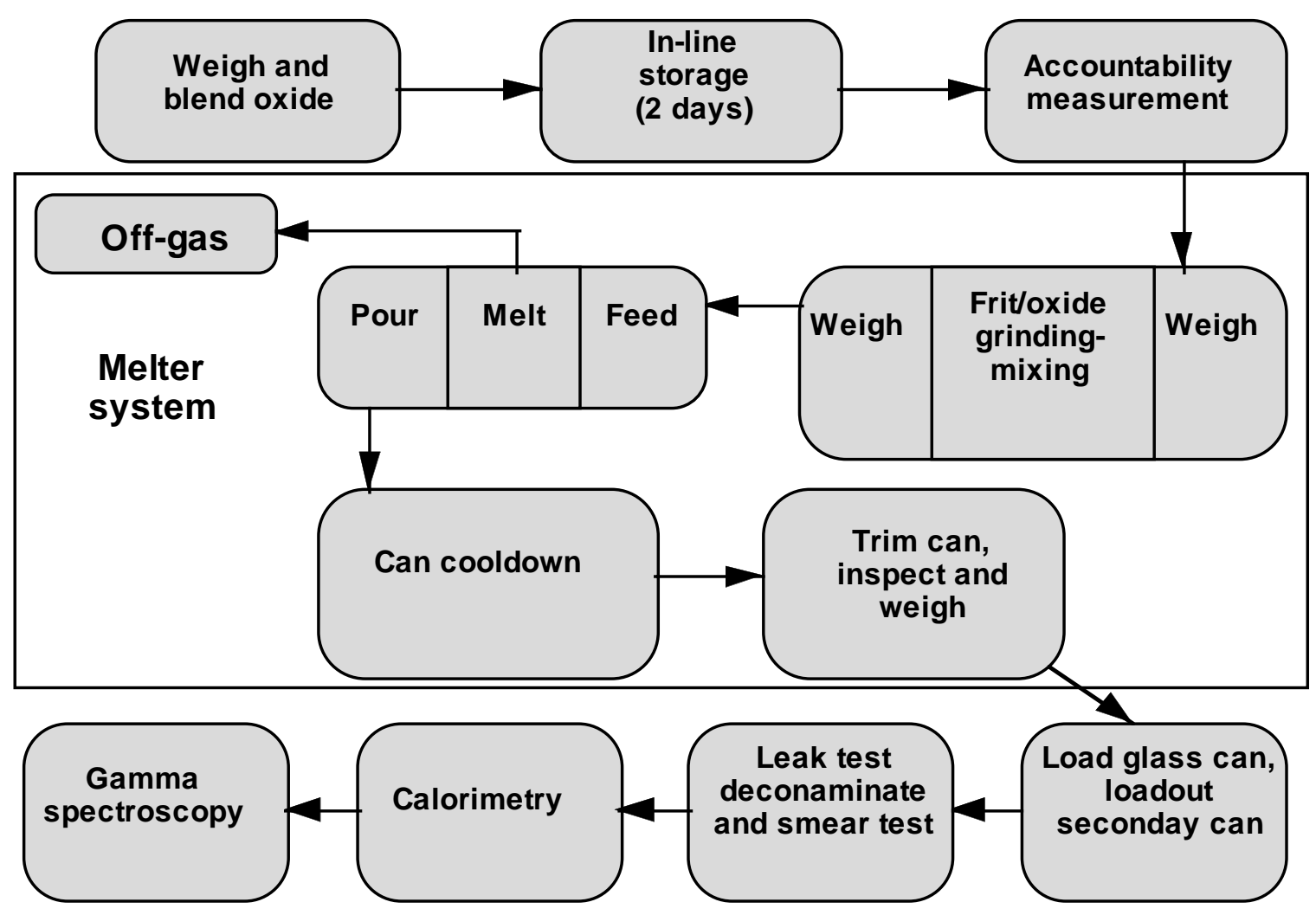

The incoming baseline feed consists of the $\mathrm{PuO}_{2}$ powder admixed with incoming oxidized uranium feeds in the form of mixed $\mathrm{UO}_{3}, \mathrm{U}_{3} \mathrm{O}_{8^{\prime}}$ and $\mathrm{UO}_{2}$. The plutonium oxide comes from the impure plutonium conversion head-end common to both glass and ceramic forms and, for the 50-MT case, from the separate pit disassembly and conversion plant. These oxide materials are comilled with the prefabricated, prefused LaBS frit in an attritor mill. The $\mathrm{PuO}_{2}$ $/ \mathrm{UO}_{\mathrm{x}} /$ frit mixture is then milled to the baseline 20 microns to enhance solubility of the $\mathrm{PuO}_{2} / \mathrm{UO}_{x}$ feed in the melt. Currently, it is believed that granulation will not be needed for dust control; if granulation is necessary, the binder additive must be compatible with the melter materials (e.g., no organics).

The co-ground frit and $\mathrm{PuO}_{2} / \mathrm{UO}_{\mathrm{x}}$ feed are fed directly to seven melters via a screw conveyer within a hard-piped closed system to eliminate dusting of silica and plutonium/uranium oxides. The melter, illustrated in Figure 5, is an induction-heated cylindrical ZGS (zirconia-grain stabilized) platinum-rhodium alloy vessel containing a platinum-rhodium agitator rod to enhance mixing and ensure homogeneity. Melt temperature is $1500^{\circ} \mathrm{C}$. The melter and associated equipment comprise a complex system made up of the following major 
components: the melter and drain tube, melter and drain tube induction heating systems, feed system, off-gas system, and control system. The glass feed batch size will be about $15 \mathrm{~kg}$. The cycle time is 8 hours in the melter followed by an 8 to 16 hour cooldown of the can.

Figure 5. Conceptual drawing of baseline melter.

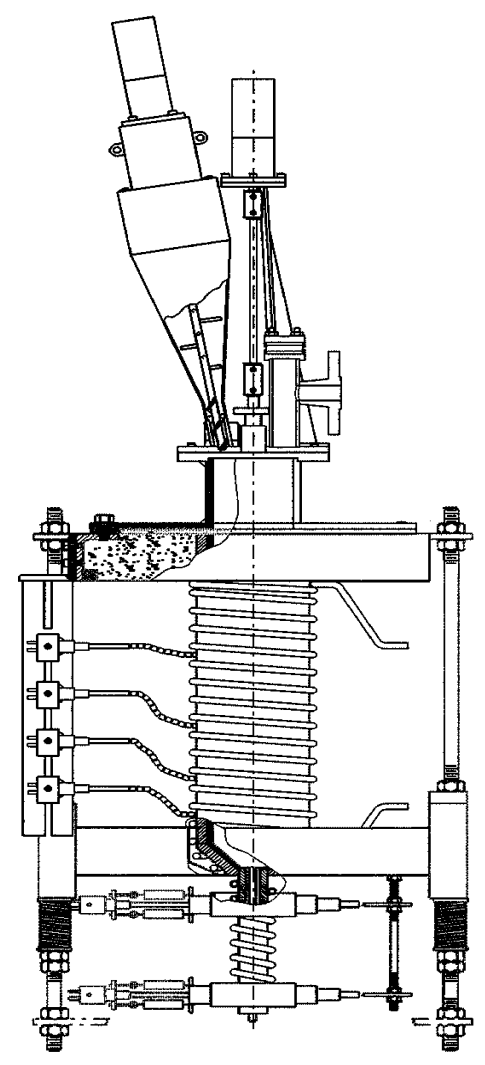

The melter and drain tube are heated with separate induction systems. The average time to start a pour is 80 seconds. The average time to stop a pour varies with the amount of glass in the melter, but is on the order of 10 to 60 seconds. The melter may have a sloped bottom so that, when the melter is drained, no glass remains on the bottom. The glass exits through the drain tube and the pour is stopped and started by cooling the pour tube with air jets. An additional feature of the agitator, is that it also doubles as a glass-pour emergency-shutoff valve by lowering the shaft into the drain-tube.

After pouring and cooling, the cans are trimmed with a commercially available pipe cutter above the meniscus. Each can containing plutonium-glass is then placed within an outer can (which protrudes outside the glove box). The outer can is sealed and detached from the glove box via a bagless transfer system. After the double plutonium-glass cans have been removed from the glove box, they are temporarily stored until ready for the canister loading step. Sixteen plutonium-glass cans are subsequently loaded into frames for insertion into empty DWPF canisters. This operation is done remotely behind 3- to 5-ft. thick 
walls. The canister containing the cans of plutonium-glass (Figure 6) is remotely placed in a shielded box on a flatbed trailer for transport to the DWPF. Upon arrival at the DWPF, a monorail crane is used to remove the canister from the shipping container and place it in another shielded container for storage in a secure vault.

Figure 6. Glass can-in-canister configuration.

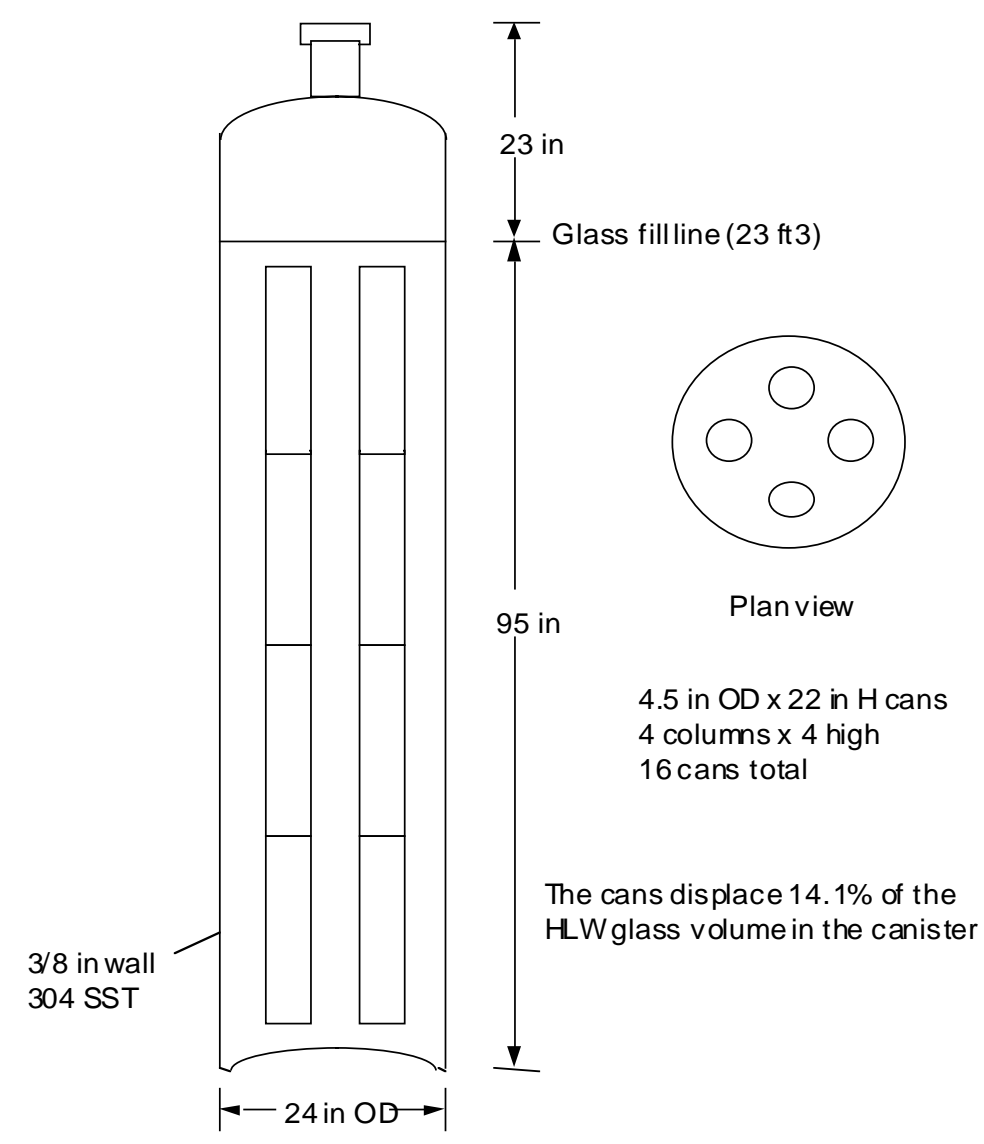

The proposed baseline process described above for the glass form does not currently have provisions for the recycle of failed melter pours. Because it is impossible to assure no glass pour or melter failures, provisions for recycle with remotely operated equipment (such as a cutting device and/or a special melting furnace) will be needed. 


\subsection{Ceramic Form and Production Process}

\subsubsection{Baseline Ceramic Composition and Physical Properties}

The baseline ceramic formulation is a phase assemblage dominated by the cubic titanate phase pyrochlore. There are three main titanate phases in the ceramic form: pyrochlore, zirconolite, and brannerite - as well as a small amount of rutile. All of these phases are acceptable host phases for plutonium and uranium. They are durable and will easily accommodate wide ranges of plutonium and uranium $(\geq 30 \mathrm{wt} \%)$, and sufficient neutron absorber elements (hafnium and gadolinium). As discussed in the TEP report (Ref. 11), the baseline ceramic form readily accommodates the range of expected impurities, either in the primary phases (pyrochlore and zirconolite) or in secondary and tertiary phases. Any additional phases that are formed from the feed impurities, are present at a maximum of a few percent and contain little, if any, actinides. The elemental composition for each of the major phases is shown in Table 7.

Table 7. Approximate elemental compositions of the coexisting phases in the ceramic form (From compilation of microprobe data).

\begin{tabular}{|l|l|l|l|l|l|l|l|l|l|}
\hline Phase & $\mathrm{Al}$ & $\mathrm{Ca}$ & $\mathrm{Gd}$ & $\mathrm{Hf}$ & $\mathrm{U}$ & $\mathrm{Pu}$ & $\mathrm{Ti}$ & $\begin{array}{l}\text { Sum } \\
\text { of } \\
\text { Metal } \\
\mathrm{s}\end{array}$ & $\begin{array}{l}\text { O } \\
\text { Equi- } \\
\text { valent }\end{array}$ \\
\hline Pyrochlore & 0.10 & 0.85 & 0.22 & 0.27 & 0.44 & 0.22 & 1.90 & 4.00 & 6.99 \\
\hline Zirconolite & 0.28 & 0.72 & 0.15 & 0.80 & 0.16 & 0.12 & 1.77 & 4.00 & 7.07 \\
\hline Branerite & 0.05 & 0.06 & 0.10 & 0.12 & 0.53 & 0.24 & 1.90 & 3.00 & 5.87 \\
\hline Rutile & 0.01 & & & 0.09 & 0.01 & & 0.89 & 1.00 & 2.00 \\
\hline
\end{tabular}

The pyrochlore-rich titanate ceramic was chosen for plutonium immobilization to ensure high loadings of plutonium, uranium, and the neutron absorbers (gadolinium and hafnium). The neutron absorbers are present to ensure criticality control in the repository. High loadings of ${ }^{238} U$ (natural or depleted uranium is added as part of the precursor oxides) are intended to provide additional criticality control in the repository over the long term through the limitation of the ${ }^{235} \mathrm{U} /{ }^{238} \mathrm{U}$ ratio as ${ }^{239} \mathrm{Pu}$ decays to ${ }^{235} \mathrm{U}$. Plutonium and uranium are interchangeable in pyrochlore.

The baseline precursor composition consists of $55.7 \mathrm{wt} \% \mathrm{TiO}_{2}, 16.5 \mathrm{wt} \% \mathrm{HfO}_{2}$, $15.4 \mathrm{wt} \% \mathrm{CaO}$ and $12.4 \mathrm{wt} \% \mathrm{Gd}_{2} \mathrm{O}_{3}$. This precursor is common to both baseline feed cases and to the variants discussed in Section 4.4. In each baseline case, 11.9 $\mathrm{wt} \% \mathrm{PuO}_{2}$ (10.5 wt \% plutonium) and $23.7 \mathrm{wt} \% \mathrm{UO}_{2}$ (20.9 $\mathrm{wt} \%$ uranium) are added to the precursor to make the overall mix. The two baseline cases differ in the relative amounts of impurities in the feed streams. 
Variations in the relative abundances of the product phases can be minimized by tailoring the composition of the ceramic precursor to specific impurities in the feed stream. The ability of the baseline ceramic formulation to utilize a single precursor for the various anticipated feeds was demonstrated by successfully testing various representative categories of the plutonium feed streams. Results of these tests are discussed in Reference 11. Because the $\mathrm{PuO}_{2}$ feeds are blended before they are immobilized, the composition of the $\mathrm{PuO}_{2}$ feed is sufficiently consistent that tailoring the precursor composition is unnecessary. Thus, a single ceramic precursor composition is used for both baseline cases.

In the ceramic immobilization process, $\mathrm{PuO}_{2}$ and $\mathrm{UO}_{2}$ are size-reduced and blended with ceramic precursors, titanium, hafnium, calcium and gadolinium oxides $\left(\mathrm{TiO}_{2}, \mathrm{HfO}_{2}, \mathrm{CaO}, \mathrm{Gd}_{2} \mathrm{O}_{3}\right)$, to make an overall mixture. The mixture is then pressed into disks at around 2,000 psi. The green disks are then reactively sintered at $1350{ }^{\circ} \mathrm{C}$ in argon for 4 hours. After sintering, the plutonium-bearing ceramic product achieves an after-fired density of $5.5 \mathrm{gm} / \mathrm{cm}^{3}$ with phases of pyrochlore $(90 \mathrm{wt} \%)$, brannerite $(5 \mathrm{wt} \%)$, rutile $(5 \mathrm{wt} \%)$, and less than $1 \mathrm{wt} \%$ plutonium-uranium-rich oxide. When impurities are added to the feed streams, the zirconolite phase (polyform of pyrochlore) is formed in significant amounts (up to $30 \mathrm{wt} \%$ ).

Tests with different ranges of particle sizes for the plutonium feed materials (Ref. 11) showed that "high-fired" $\mathrm{PuO}_{2}$ and $\mathrm{UO}_{2}$ with particle sizes $<20 \mu \mathrm{m}$ are completely reacted with the ceramic matrix by sintering at $\geq 1350^{\circ} \mathrm{C}$ for four hours. In the baseline formulation, it is assumed that uranium is added as $\mathrm{UO}_{2}$ and not $\mathrm{U}_{3} \mathrm{O}_{8}$ or $\mathrm{UO}_{3}$ and that the pellets are sintered in argon. Tests also demonstrated that the pellets can be sintered in air if uranium is added as $\mathrm{UO}_{2}$ and that uranium can be added as $\mathrm{U}_{3} \mathrm{O}_{8}$ if the pellets are sintered in argon.

\subsubsection{Baseline Ceramic Process}

The baseline ceramic process (less the feed conversion and preparation headend operations common to both glass and ceramic processes) is depicted schematically in Figure 6 . The production process operates in a batch mode to facilitate material accountability and avoid criticality. The non-radioactive plant feeds are all stored outside the ceramic processing facility. The ceramic precursor, calcined at $750^{\circ} \mathrm{C}$ for 1 hour, is supplied by a vendor to specifications on powder size, chemistry and homogeneity. All operations are performed in shielded gloveboxes similar to those in MOX fuel fabrication facilities. Most material handling are performed with automated, hands-off equipment.

The incoming plutonium feed materials (from the impure plutonium feed conversion headend and the pit/disassembly and conversion plant located elsewhere) are co-milled with the incoming $\mathrm{UO}_{2}$ in a dry state and in a highspeed attrition mill. The $\mathrm{PuO}_{2} / \mathrm{UO}_{2}$ mixture is added to a blender with ceramic precursors and the material homogenized. This mixture is transferred to a 
granulator where a binder and water mixture will be added to agglomerate the material. The agglomerated material is dried and stored until added as a free flowing powder to dies in a double piston hydraulic press. The powder is then pressed at around 2,000 psi. After inspection, approximately 80 "green" disks are collected on trays, loaded into a sintering oven and sintered at $1350^{\circ} \mathrm{C}$ in argon for 4 hours.

Figure 7. Ceramic process flowsheet.

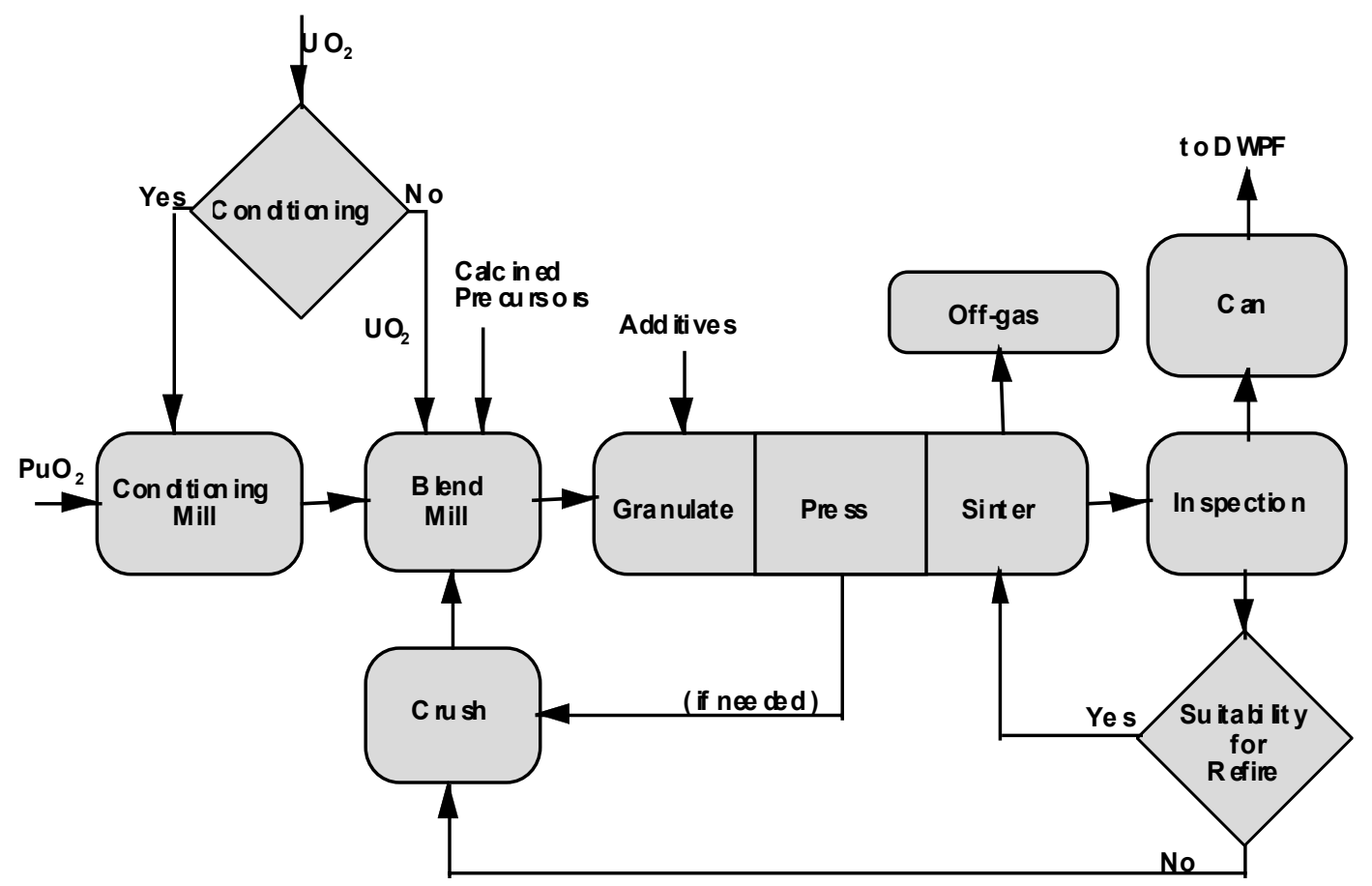

After sintering, the ceramic disks are removed from the oven, inspected, and loaded into a "bagless transfer" can. The can is sealed and removed from the glove box via a bagless transfer system (basically the same system and operations described for glass). After removal from the glove-box line, the ceramiccontaining cans are stored until they are ready for canister loading. Twenty eight of these cans are loaded into frames, which are then inserted into an empty DWPF canister. The remaining handling and transfer steps are the same as for the glass forms (Section 4.2.2). The major processing parameters for the baseline ceramic form are summarized in Table 8. 
Figure 8. Ceramic can-in-canister configuration.

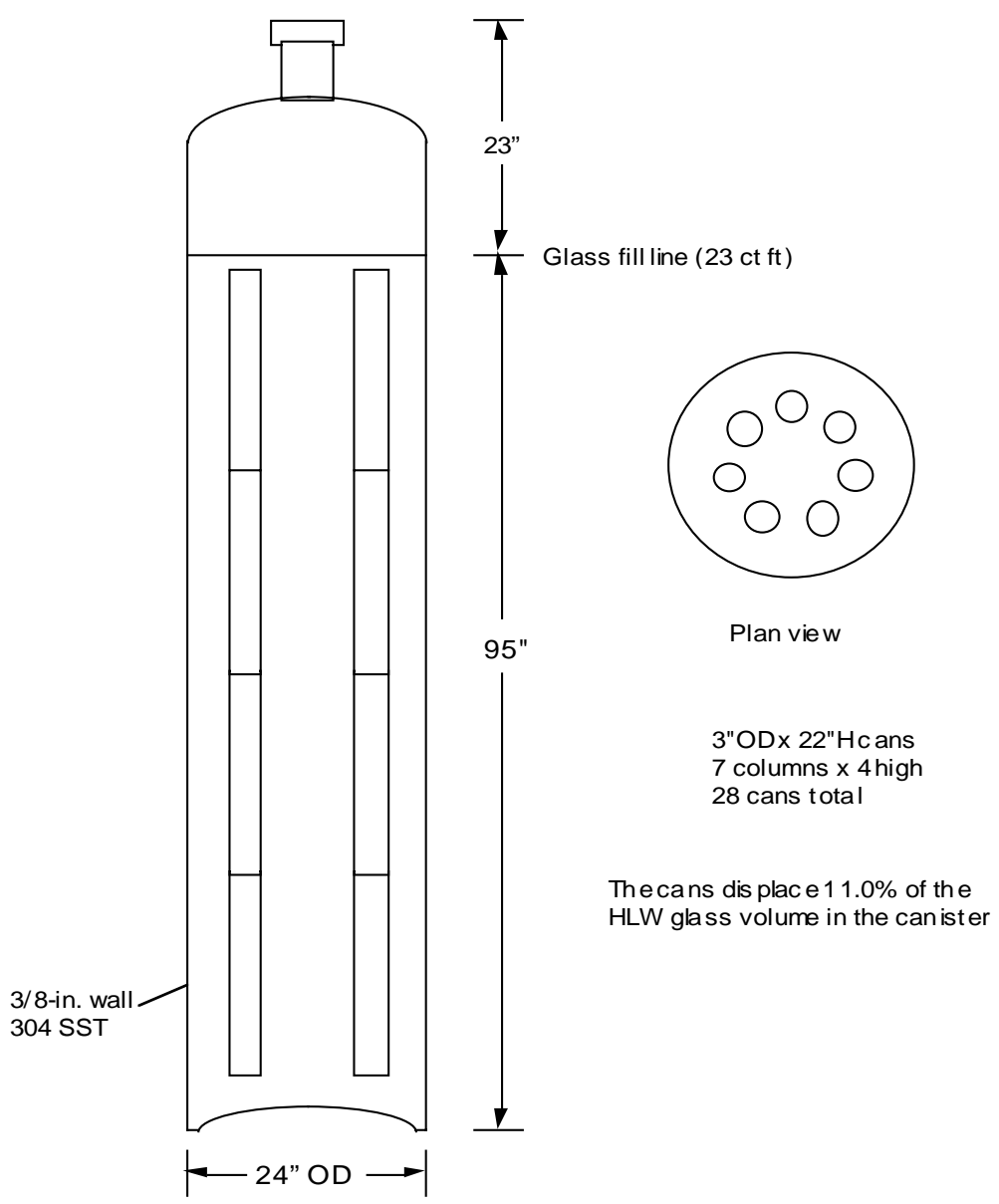

The baseline ceramic process flowsheet has provisions for recycling unacceptable material after inspections of the green pellets and after sintering. Recycling of sintered pellets is considered to be a rare occurrence because the key inspections on product homogeneity and reproducibility are performed during the green pellet inspection. Careful control of the conditions used in milling, blending, and granulating ensures ceramic feed powder and pressed pellets of consistently high quality, resulting in similar consistency in the sintered products. 
Table 8. Major processing parameters of the 50 MT Pu and the 17 MT Pu baseline processes.

$\begin{array}{ll}\text { Batch size } & 39.7 \mathrm{~kg} \\ \text { Batch contents } & 25.5 \mathrm{~kg} \text { of ceramic precursor } \\ & 4.167 \mathrm{~kg} \mathrm{Pu}\left(4.727 \mathrm{PuO}_{2} \mathrm{~kg}\right) \\ & 8.333 \mathrm{~kg} \mathrm{U}\left(9.453 \mathrm{UO}_{2} \mathrm{~kg}\right) \\ \text { Pellet diameter } & 2.65 \mathrm{in} \\ \text { Pellet height } & 1.00 \mathrm{in} \\ \text { Pellets/batch } & 80 \\ \text { Pu/pellet } & 52.1 \mathrm{gm} \mathrm{Pu}(59.1 \mathrm{gm} \mathrm{PuO}) \\ \text { Pellets/can } & 20 \\ \text { Pu/can } & 1.04 \mathrm{~kg} \mathrm{Pu}(1.18 \mathrm{~kg} \mathrm{PuO}) \\ \text { Cans/batch } & 4 \\ \text { Can diameter } & 3 \text { in O.D and } 2.75 \mathrm{in} \mathrm{I.D.} \\ \text { Can height } & 21 \mathrm{in} \\ \text { Number of batch/day } & 6(25 \mathrm{~kg} \mathrm{Pu} \text { or } 28.4 \mathrm{kgPuO}) \\ \text { No. of sinter furnaces } & 6 \\ \text { Annual production } & 5.0 \mathrm{MT} \mathrm{Pu} \text { in } 3 \text { shifts at } 200 \text { days/year } \\ & 47.6 \mathrm{MT} \text { of ceramic/year }\end{array}$

\subsection{Alternatives to the Baselines}

Variants to the baseline forms and to the can-in-canister design may offer advantages over the baseline forms for some evaluation criteria. These variants are briefly described here.

\subsubsection{Alternative Glass and Ceramic Forms}

The alternative glass and ceramic formulations (properties summarized in Table 4) are capable of immobilizing higher concentrations of plutonium for both baseline feed cases.

For the glass form, the plutonium loading would be increased from $8.8 \mathrm{wt} \%$ plutonium to about $10.6 \mathrm{wt} \%$ plutonium, or possibly greater. The plutonium concentration limit is restricted by the solubility limit of the oxide in the glass at $1500^{\circ} \mathrm{C}$. As the plutonium concentration is increased beyond the baseline value, some fraction of the $\mathrm{PuO}_{2}$ will crystallize in the melt, forming fine- grained particles. Evidence presented to the TEP indicates that these crystalline oxides may also contain hafnium (Ref. 11).

For the ceramic form, the plutonium loading would be increased from $10.5 \mathrm{wt} \%$ plutonium to $15.7 \mathrm{wt} \%$ plutonium for the $50 \mathrm{MT}$ case, and from $10.5 \mathrm{wt} \%$ plutonium to $16.2 \mathrm{wt} \%$ plutonium for the $17 \mathrm{MT}$ case. The total actinide content would be maintained at about $30 \mathrm{wt} \%$, which determines the amount of $\mathrm{UO}_{2}$ additive needed. There would be no change in the phase assemblages formed upon sintering and no change in the processing steps. 
The higher concentrations of plutonium in the alternative forms allow more plutonium to be immobilized per DWPF canister, reducing the number of DWPF canisters affected and the number of additional HLW canisters to compensate for the volume occupied by the plutonium forms. This would save about $\$ 500,000$ per canister avoided.

\subsubsection{Can-in-Canister Configuration Options}

Several alternative concepts for the internal form configuration within the canister are being considered to enhance the proliferation resistance of the immobilized plutonium. These would be deployed, only if judged necessary, to thwart at least one approach by a terrorist organization to extract the plutoniumbearing forms (see Section 5.2). The simplest of these concepts would involve welding the plutonium form cans to each other and to the supporting frame. Another, more robust, concept would involves reinforcing the plutoniumcontaining cans with either steel rebar or armor. This second approach, with the additional metal components, could cause the DWPF glass to cool faster, which may, in turn, inhibit the HLW glass from intimately surrounding the plutonium containing can or from fully filling the canister. Any such design would require large-scale testing.

Another approach involves concepts that allow the HLW glass to intimately contact the plutonium forms. This could be accomplished by the use of friable cans that would come apart in the molten glass or by placing small ceramic pellets or glass marbles containing the plutonium into wire baskets that could be suspended within the DWPF canister. Small-scale tests with surrogate glass indicate that the ceramic forms would not be chemically affected by their direct contact with the molten HLW glass. Similar tests with the LaBS glass indicate a slight surface reaction. When subjected directly to the molten HLW glass temperature, some plutonium oxide might precipitate from the amorphous phase, depending on the plutonium concentration in the LaBS glass.

These alternative concepts would essentially eliminate concerns about terrorists destroying the outer immobilization canister and carrying away the canned plutonium forms. 


\subsection{Assessment of Forms Against Criteria}

The TEP report (Ref. 11) provides an evaluation of the two candidate technologies against the individual decision criteria and metrics without regard to the relative importance of these factors. The purpose of the assessment presented here is twofold: (1) to distill and clarify the important discriminating results from the TEP report and (2) to qualitatively rate the two forms for each of the decision factors, where supported by the data and available analyses (i.e. those provided in the TEP report and in presentation materials and discussions during the TEP meeting). In Section 6.0, an overall assessment is made that accounts for the relative weights of the criteria.

The discussion in this section focuses on those decision factors for which there is a potential difference between the two technologies. Where there were no discernible differences found by the TEP and this assessment, the fact that neither form has an advantage is noted but not elaborated upon. The TEP evaluation and this assessment, to a significant extent, had to rely on qualitative analysis. In some cases, quantitative information was available, although an interpretation of the "raw" data for the specific metrics was still required. Most of the metrics required analyses that took into account a variety of non-quantified information, which, in turn required significant technical judgment. Where differences of opinion occurred between this assessment and the findings or interpretations in the TEP report, the rationale for the differing viewpoints is provided. Where this assessment agreed with the findings in the TEP report, the results are summarized and converted to a relative rating.

\subsection{Ratings}

A simple grading system was adopted to compare the relative worths of the two forms for each decision factor. The grading system has two parts: (1) a comparative rating of the two forms for the factor or metric and (2) the relative importance of the factor or metric. The following simple qualitative scale was devised to grade the advantage of the better form over the inferior form for each factor:

- No discernible difference (ND): the two forms were judged to be equal for the factor

- Insignificant difference (I): there is a discernible difference between the two forms, but it is too small to be significant, or there are sufficient other mitigating considerations to render the stated or measured difference insignificant

- Small advantage (S): the better form has a small, but still significant advantage of achieving the metric's stated or implied goal. Example: a cost difference of " $\mathrm{S}$ " indicates that the worse form is expected to cost $\$ 25$ million to $\$ 100$ million more than the better form. 
- Medium advantage (M): the better form has a moderately significant advantage of achieving the metric's stated or implied goal. Example: the worst form would cost between $\$ 100$ million to $\$ 200$ million more than the better form.

- Large advantage $(\mathrm{L})$ : the better form is much superior in achieving the stated or implied goal, or the worse form has significant difficulty in meeting the goal. Example: the worse form is expected to cost $>\$ 200$ million more than the better form.

A " $G$ " or " $C$ " code was used to indicate whether the advantage was assigned to glass or ceramic, respectively. DOE-MD specified weights for each criterion to provide a measure of the relative importance of the criterion. These criterion weights were allocated by LLNL to factors within each criterion. The factor weights are considered in Section 6 and Appendix A.

\subsection{Resistance to Theft and Diversion}

The objective associated with this criterion is to provide for comprehensive control and protection, external and intrinsic, of the plutonium. A parallel study of the non-proliferation effectiveness of the can-in-canister immobilization approach provided input for both the TEP report and this assessment for Criteria 1 and 2 (Ref. 21).

There are four applicable factors under this criterion that could discriminate between the glass and ceramic technologies (1a, -c. -d, and -e; Table 1). A fifth factor, minimization of transportation, facilities, and sites, was not a discriminator for choosing between the two technologies. The ratings for the four applicable factors are generally consistent with the TEP findings:

5.2.1 Low Inherent Attractiveness: I (G) - Insignificant advantage for glass. The TEP report recognized the slight difference in plutonium concentrations for the glass and ceramic forms ( $8.8 \mathrm{wt} \%$ and $10.5 \mathrm{wt} \%$, respectively) during the feed conditioning and forming steps, and the slightly higher canister inventory for the ceramic form ( $29 \mathrm{~kg}$ vs. $21 \mathrm{~kg}$ ). However, the quantities and forms of both immobilization forms are within the same DOE attractiveness category (DOE Order 5633.3B). Furthermore, these differences are mitigated by the stringent safeguard controls and facility / site security systems through the final HLW glass immobilization step. The draft TEP report also pointed out that the higher neutron dose associated with the glass form could make the material less attractive for diversion. However, this is a more important issue for achieving worker exposure that is as low as reasonably achievable (ALARA), and is not a significant deterrent against diversion (dose is discussed under the ES\&H criterion, Section 5.5). Therefore, from a practical standpoint, the two processes have comparable levels of attractiveness throughout the immobilization processing system. 
5.2.2 Minimizing Processing Steps and Complexity: ND - The processing systems for both glass and ceramic technologies are essentially comparable with respect to complexity as related to opportunities for diversion.

5.2.3 Safeguards and Security Assurance: S (C) - Small advantage for ceramic due to its more developed and easier-to-achieve material accountancy. Two metrics are considered for this factor, measurement uncertainty and accessibility. Regarding the first, accountancy for the ceramic materials involves the straightforward application of standard NDA techniques that are applied in the MOX fuel industry (e.g., weighing, gamma and neutron spectroscopy, calorimetry and piece counting). For glass, standard NDA techniques are used to the point where the feed is mixed with the frit material. Subsequently, a combination of process control modeling based on feed analyses (similar to that used for the DWPF), sampling and analysis, and can weighing is used. This approach has not been developed and validated for plutonium systems, but should not be a fundamental problem. NDA may be complicated by the high neutron field from the $(\alpha, n)$ reaction in boron, a major constituent in the LaBS glass.

The other measure for this factor is the accessibility of the plutonium to possible diversion, considering a combination of packaging, access, and special equipment needed to handle the plutonium materials. According to the TEP report, glass has a slight advantage for this measure; ceramic pellets would be easier to divert than cans of glass due to their smaller size and lower radiation signature. This advantage is insignificant however, because the processing operations for both would be conducted in a fully secured, safeguarded, and automated plutonium glove box line. A missing ceramic pellet would be easily and quickly detected. Furthermore, it would take many ceramic pellets to provide the plutonium contained in a single can of plutonium glass, and at least quadruple that number to yield a significant quantity of plutonium.

\subsubsection{Difficulty of Retrieval and Extraction by Rogue Party: S - M (C) - Small to} moderate advantage for ceramic as a consequence of the higher degree of difficulty in the processes needed to separate plutonium from the ceramic form compared to the glass form. The assessment for this factor considered the following:

- The standard for sufficiency is the Spent Fuel Standard; i.e., are retrieval and extraction from the two can-in-canister form variants as difficult as for spent fuel?

- The difficulty of retrieving a sufficient quantity of plutonium.

- The difficulty of extracting a sufficient amount of pure plutonium from the stolen forms to fabricate a weapon.

- The detectability of the recovery activities. 
A major threat of theft would be associated with the transport of the HLW packages containing can-in-canister immobilization forms to the repository. An earlier study by an expert panel on proliferation (Red Team Evaluation; Ref. 22) identified one scenario for which the can-in-canister form might be more vulnerable than spent fuel. This scenario involves interdiction by a terrorist group of a HLW package shipment, forced entry into the shipping cask, removal of a canister containing plutonium forms, accessing and removing individual cans of the plutonium forms, and escaping using a helicopter. The major issue for the can-in-canister immobilization package involves the fact that the plutonium form itself contains no fission products and thus its separation from the surrounding HLW glass could be accomplished without heavy equipment or shielding.

A follow-on study to the "Red Team" report on the proliferation resistance of the can-in-canister form is being performed by the immobilization development ("White") team. An early draft report from that study provides these key findings (Ref. 21):

- Both the glass and ceramic can-in-canister forms meet the Spent Fuel Standard based on the degree of difficulty, time, and cost for recovering plutonium.

- Several design modifications to the original can-in-canister concept could be made that would effectively retard or preclude entry and access to the plutonium cans for both the glass and ceramic forms. These include mechanical enhancements, such as welding the cans to the rack and armoring, and designs that would allow the HLW glass to intimately contact the glass or ceramic forms (Section 4.4).

- The ceramic form has an advantage over the glass form with respect to the difficulty of plutonium recovery.

Recovery from glass can be achieved with a modification to an existing (published) plutonium recovery process. The ceramic form, on the other hand, would require a more chemically complex process that has not been developed for plutonium-bearing titanate minerals. The "White Team" draft report discusses preliminary process flowsheets and equipment concepts, and provides rough cost and time estimates for recovery by a terrorist organization or rogue nation (Table 8). Preparation time, recovery time, and costs are greater for the 
Table 9. Summary comparison of ceramic and glass plutonium immobilization forms for criterion I (resistance to theft or diversion of two or more significant quantities by unauthorized parties).

1. Time to reprocess

a. Development time

b. Design, procurement and construction time

c. Process equipment

d. Actual processing time

2. Process Parameters
a. U/Pu ratio
b. Processing steps required
c. Anticipated yield (normalized)

3. Cost to reprocess

4. Detectability of reprocessing activities

5. Separability from radiation barrier
Ceramic Form

6 to 12 months depending upon the level of expertise

10 to 42 months

Specialized

4 to 14 weeks

2 for 50 MT case
2 for 17 MT case
14 for minimal yield
18 for higher yield
1.0 to 3.3

Within capability

Purchase of specialized equipment and process chemicals

Feasible
Glass Form

Documented in open

literature

6 to 30 months

Piratable from many small industries

3 to 12 weeks

0.3 for $50 \mathrm{MT}$ case

0.95 for 17 MT case

12

2.7 to 3.8

Within capability

Purchase of process chemicals

Feasible
Spent Fuel

Documented in open literature

6 to 30 months

Piratable from many small industries

3 to 12 weeks

$>19$

11

$15.7 \pm 7.9$

Within capability

Purchase of process chemicals

Feasible 
ceramic form than for glass, but not by large margins. In addition, the chance of failure (or partial recovery) in the ceramic recovery process - especially by an organization that has not performed lab-scale developmental tests of this new process with plutonium - is greater than for recovery from glass. Finally, the longer installation and processing times for the ceramic form combined with the need for special equipment offer a slightly greater opportunity for the terrorist organization to be discovered.

\subsection{Resistance of Retrieval, Extraction, and Reuse by the Host Nation}

There are two factors for this criterion: (1) cost and time of retrieval and recovery, and (2) assurance of detection. The latter factor has a much lower weight (Table 1) and is not a discriminator because detection of actions to recover plutonium on a large scale from either form would be relatively easy.

Difficulty, Cost, and Time for Recovery: I (C) - Unlike the similar factor for Criteria 1 , there is only an insignificant advantage for the ceramic form because the extensive resources of the host nation would overcome the processing difficulty advantage for ceramic. As for the case of a terrorist organization or rogue nation, an analysis of the cost and time required for the host nation to recover metric-ton quantities of plutonium from the immobilization form was performed by the "White Team." The F-Canyon facility at SRS was used to assess the equipment configurations and modifications that would be required to recover large quantities of plutonium from can-in-canister forms containing glass or ceramic. Given the large resources and likely resolve of the host nation (i.e., enough to break treaty agreements), the higher difficulty, time, and cost to recover plutonium from ceramic forms versus glass forms is believed to be insignificant. For the host nation, recovery of plutonium from either of the can-in-canister forms would be comparably as difficult as recovery of plutonium from spent nuclear fuel.

\subsection{Technical Viability: Repository Acceptability}

Two of the factors under Technical Viability - Technical Maturity and Viability Risk are assessed relative to their impacts on cost and timeliness (Criteria 5 and 6) in Sections 5.6 and 5.7, as requested by MD (Ref. 17). In this section, a rating of the two technologies for these factors is provided with minimal discussion. The major factor considered under Technical Viability is Repository Acceptability of the disposal form.

Technical Maturity and Viability Risk: ND - The TEP and this evaluation found no significant difference between the two technologies with respect to their overall maturity. Glass has a small advantage over ceramic in the area of product knowledge and control. The ceramic form needs additional testing to verify its ability to produce acceptable products across the expected range of feed 
materials. This glass advantage is offset by the lower maturity of the hightemperature induction-heated melter system for glass, compared to the very mature MOX fabrication technology being adapted for the ceramic form. Neither of these issues is believed to be a potential "show stopper" or to represent a significant risk to the immobilization project.

Repository Acceptability of Disposal Form, S - M (C) - Small to moderate advantage to the ceramic form due to its expected higher durability under repository conditions and its lower potential for long term criticality. This factor is composed of several metrics, principally: dissolution or corrosion rate, expected surface area, and effects of radiation damage (from $\alpha$ decay). The major performance issue is the ability of the candidate forms to provide assurance against a long term criticality event in the repository.

The TEP members reviewed data presented on the LaBS glass and titanate-based ceramic forms' dissolution rates, published data on the effects of radiation on glasses and crystalline ceramic phases similar to the immobilization forms, and a TRW study (Ref. 23) of degraded-mode criticality of the glass and ceramic forms. The TEP findings were:

- Both immobilization forms should be acceptable to the repository from the standpoint of criticality both within the waste package and for a degraded waste package. Because the total radionuclide inventory in the immobilization forms would be small compared to the total projected inventory in the repository, the release of constituents from the immobilization forms is not expected to significantly affect results of the total system performance assessment (Ref. 24).

- Dissolution rates of the ceramic for matrix elements calcium and, plutonium, and the neutron absorbers hafnium and gadolinium are substantially lower (by more than two to four orders of magnitude) than their counterparts for glass (see Figures 6.4.1-6.4.4 in the TEP report).

- Radiation damage from $\alpha$-decay is expected to have minor effects on the glass form's dissolution rates. However, the ceramic form will become metamict and undergo some cracking, which is expected to increase its dissolution rate by an order of magnitude or more.

Consequently, the available data reviewed by the TEP suggest that the long term durability of the ceramic form (after it becomes metamict) should be more than an order of magnitude superior to LaBS glass. Within the TEP, there were differing viewpoints on the value of the ceramic's higher durability with respect to this criterion. Advocates of the glass form have asserted that, because borosilicate glasses have been so thoroughly studied and have predictable dissolution properties, the LaBS glass would be easier to qualify than the more durable but less studied pyrochlore-based ceramic form. However, this 
evaluation argues that higher durability is more important than familiarity in assessing the two forms with respect to this criterion.

At present, there is no directly applicable guidance for the plutonium immobilization form, but the requirements for the HLW glass are assumed to form the basis for the minimum requirements for this assessment. The immobilized form presents new issues related to criticality safety that are not of concern for the disposal of HLW, and it is possible that additional requirements or specifications will be imposed on the immobilized form. Although the performance of both forms is likely to be adequate from a repository acceptability standpoint, there is a clearly resolvable difference between them: in every leaching or dissolution test performed to date, the forward dissolution rate and elemental release rates from the ceramic were consistently lower than from the glass. The degree to which these differences have value was not assessed by the TEP.

Assessments of long-term repository performance are necessarily dependent on models because of the long time periods involved. In the present case, long-term predictions of the release of elements from a failed waste package are dependent on models for dissolution rate laws for the waste forms, the kinetics of precipitation of alteration products, the aqueous solubilities of those products, and the generation and transport of colloidal particles. The process of modeling the geochemical behavior over very long times in a repository is subject to considerable uncertainty. As a result, there will always be uncertainty as to whether the plutonium, uranium, and neutron absorbers separate after they are released from the degrading waste form. This separation may occur within a waste package, in the near-field repository environment, or in the far field. Therefore, the safest approach to assuring criticality safety is to minimize their release in the first place, i.e., to keep them sequestered in the immobilization form in the as-fabricated state.

As pointed out by the TEP, as long as the immobilized material remains in an undegraded state, there are no issues related to criticality due to the overabundance of neutron absorber in the forms. The magnitude of the dissolution or reaction rate of the forms with groundwater in the repository is the key factor controlling how long the forms will remain in this state. It is therefore prudent to build in as large a safety factor as possible, using parameters that can be controled or measured with confidence, rather than to assume that the models, input data, and boundary conditions (e.g., the time-dependent chemical and physical environment of the repository) are accurate. The forward dissolution rate, which is the maximum possible dissolution rate, is such a controllable and measurable parameter. The available data indicate that the forward dissolution rate of the ceramic is many orders of magnitude lower than that of the LaBS glass. 
The "value of information" in a repository performance assessment is a convolution of sensitivity and uncertainty; the more sensitive repository performance is to a parameter, the higher the confidence needed in that parameter value. For forms with similar radionuclide inventories but release rates differing by orders of magnitude, there is less sensitivity to the higherperforming material. The ceramic appears to perform orders of magnitude better than needed, so a higher safety margin and higher safety confidence can be achieved with fewer data. In other words, the closer to the performance limits a waste form is, the more data and greater certainty in the data and models that are required to achieve safety confidence. There is value in performance beyond the minimum. Depending on how far performance is from the minimum, the qualification and licensing process may be easier for a better performing form.

In the area of long-term criticality safety, there is an additional benefit to the reduced dissolution and release rate of the ceramic. The longer the times to release, the greater the temporal and spatial dispersion is likely to be through natural distributions of processes, and the lower the absolute concentrations at any given time and place. In addition, the added ${ }^{238} \mathrm{U}$ in the ceramic formulation provides an isotopic diluant for the decay-product ${ }^{235} \mathrm{U}$, which reduces the potential for criticality over the long term. The degree to which this offers a significant enhancement in criticality safety has not been assessed.

The process of obtaining repository acceptability is likely to be far from straightforward, based on past experience. In the absence of overriding benefits in other areas (e.g., ease of processing, better proliferation resistance, etc.) there is no reason to select an immobilization form that is simply adequate when better alternatives are available. Indeed, the environmental impact consideration process, to which the repository is subject, involves an explicit consideration of alternatives and an explanation of why they were rejected. It would be difficult to defend an argument of adequacy (glass) in the face of documented superior options (ceramic) unless there are offsetting benefits to the selected alternative.

\subsection{Environmental, Safety, and Health Compliance}

The objective of this criterion is to ensure that high standards of public and worker safety and environmental protection are achieved. There are three factors that were considered in assessing the expected performance of the two technologies with respect to this criterion: (1) public and worker health and safety; (2) waste minimization; and (3) known and manageable waste forms.

\subsubsection{Public and worker health and safety - S - M (C) - Small to medium} advantage to the ceramic technology because of its much lower potential dose rate versus that for the glass form. The assessment for this factor begins with the premise that the facility design and operations will satisfy all applicable federal statutes and regulations. As a consequence, there are few distinguishable differences between the two immobilization form processes. For the general 
metric of industrial hazards, the TEP and this assessment found that, at the present state of knowledge regarding the equipment and facility concepts for the two technologies, there appears to be no significant distinction (Ref. 25 - 28).

For the metric of radiation exposure to the public, there are no significant differences between the two technologies for both normal operations and for potential accident situations (Ref. 25 - 28).

However, for the metric of potential worker dose, there is a significant difference between the two forms. The ceramic process has a significant advantage over the glass process due to the much higher neutron radiation source strength associated with the glass form. This stems from the $(\alpha, n)$ reaction that occurs in boron, a key constituent in the LaBS glass. The high neutron rate occurs beginning with the glass frit-plutonium feed milling/blending step through canister operations prior to entry into the DWPF canyon.

To assess the potential worker dose implications of the higher neutron source rates for glass, both LLNL and WSRC calculated comparative doses for the ceramic and glass processes for various shielding configurations and distances (Refs. 29 and 30). Figure 9 summarizes the results of these calculations.

Figure 9. Total dose glass versus ceramic for various operating configurations.

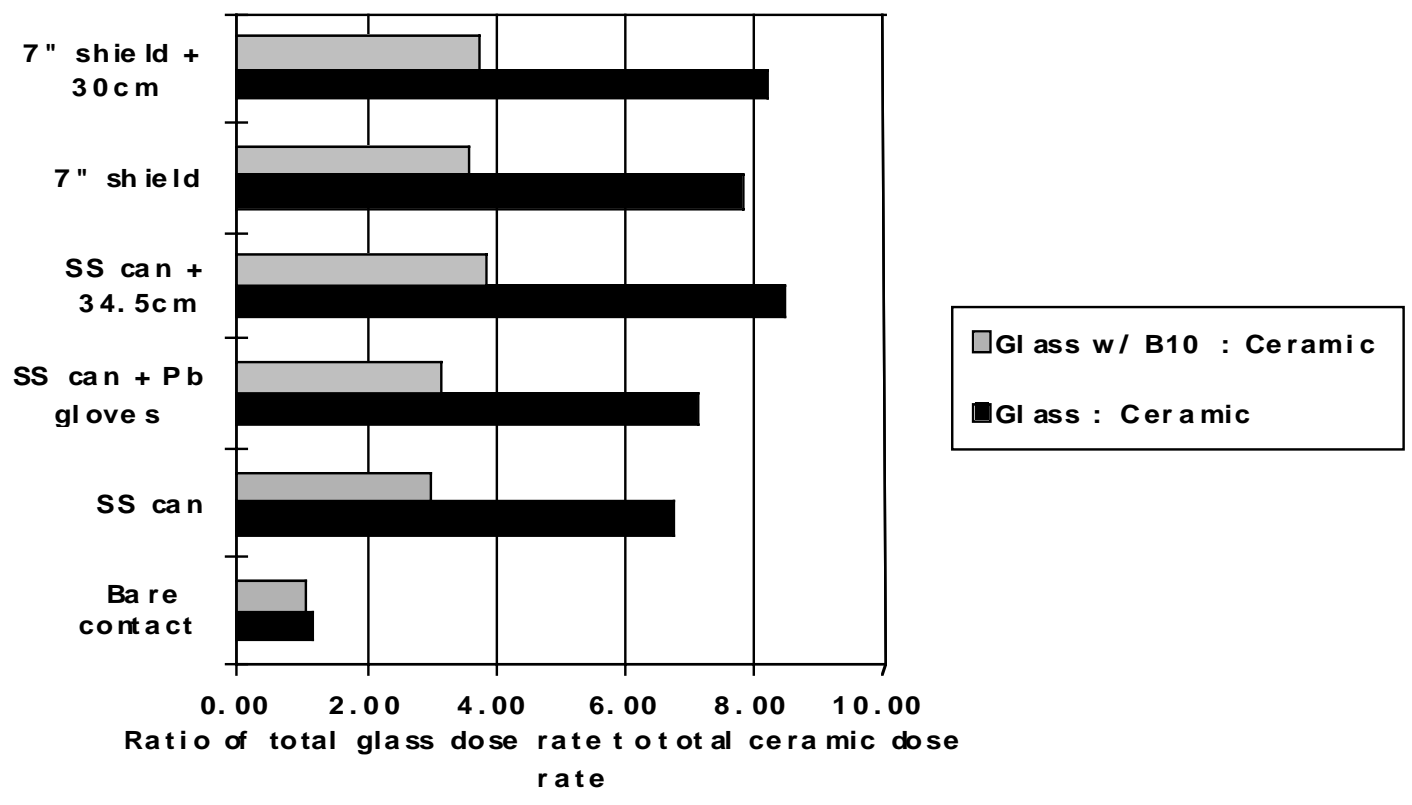


As illustrated in Figure 9, the baseline glass form generates a radiation field seven to eight times higher than the ceramic form. The radiation field is dominated by the neutron dose. If isotopically enriched boron-10 is used, the potential exposure differences would decrease to a factor of three to four times higher for glass than ceramic.

The practical implications for the higher potential dose for glass must be viewed in the context of the respective design and operating concepts for the glass and ceramic processes. In particular, the equipment systems for both forms will be mostly automated for both dose control and production efficiency. For the ceramic process and the powder-conditioning portion of the glass process, the equipment and automation techniques can be adapted from the MOX fuel industry. Even with this overall design approach, however, there are significant implications of the higher glass dose rate:

- There are a few troublesome operations that might benefit from limited operator handling, such as can extraction from the glove box (decontamination of the weld area). With the ceramic form, such "hands on" operations would be possible, as illustrated by Figure 10. For glass, such "hands on" operations would be much more restricted. This difference could translate into simpler designs for the ceramic equipment and is a potential cost impact (Section 5.6).

- The glass line after milling will generally require more shielding than the ceramic line to achieve comparable dose rates to operational and maintenance personnel. To avoid significant deinventorying of materials when maintenance operations need to be performed on a specific piece of equipment, additional shield barriers and space may be needed for the glass process. This also is a potential cost impact (Section 5.6).

- Finally, the DOE goal of "as low as reasonably achievable" (ALARA) exposure to personnel can more easily be achieved with the ceramic process. All other factors being equal, the ALARA principle favors the ceramic immobilization process. 
Figure 10. Working hours per week for canned forms for different operating conditions.

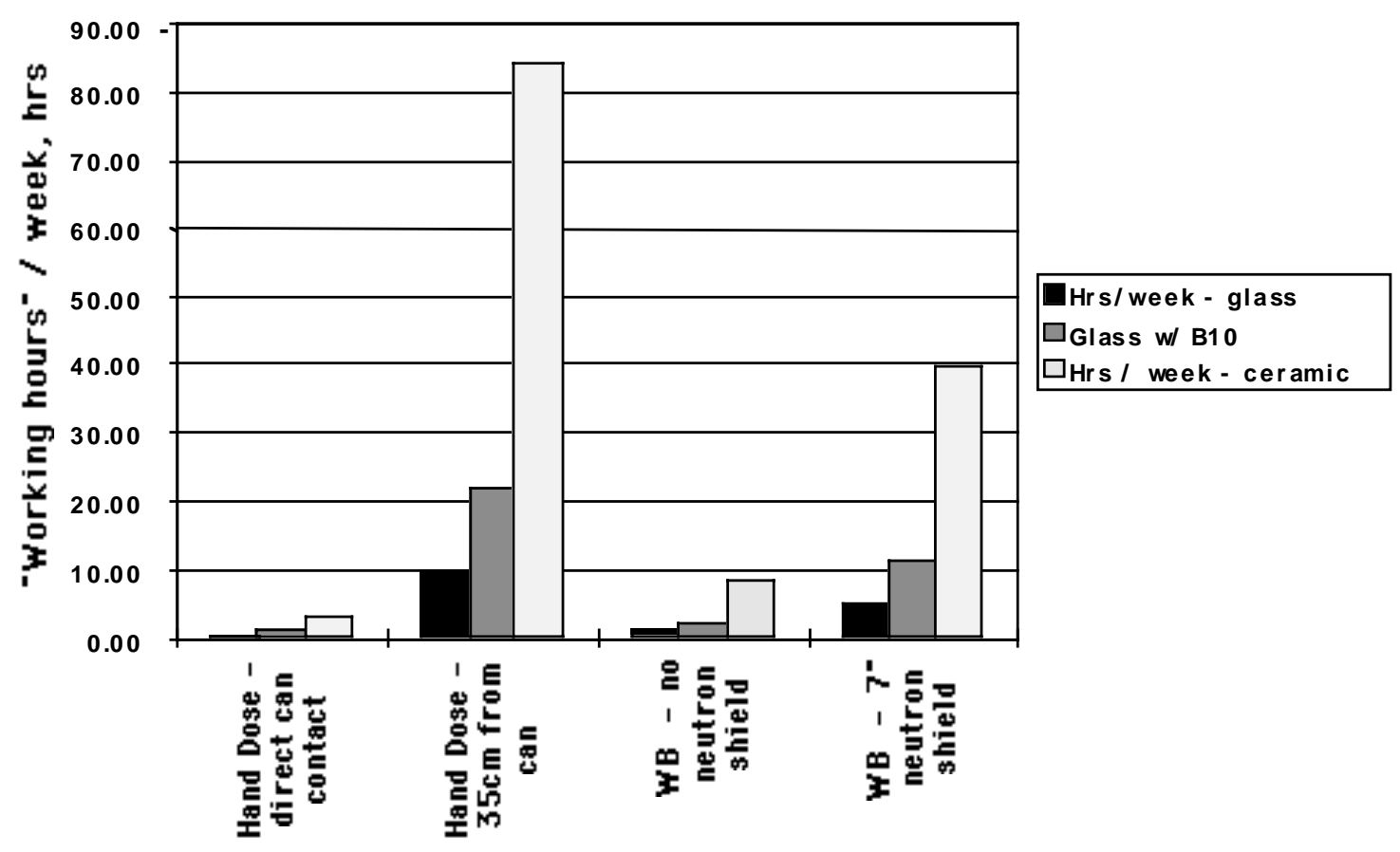

The other metric associated with public or worker exposure is contamination potential. Because both immobilization form processes use similar feed preparation and particle sizing steps in the discriminating operations, they are judged to be equivalent for this metric.

5.5.2 Waste Forms and Minimization: ND - There appears to be no significant difference in the two processes with respect to their generation of waste streams. However, in the baseline process flow diagram for glass (Figure 4), there is no provision for recycling plutonium-containing glass from failed melter runs. A recycling capability is needed, because it is impossible to ensure against a failed melter or melter pour. If there is no recycling for glass, then the baseline process would have a problematic waste stream and in turn this metric would rate a moderate advantage for the ceramic process. The "no difference" rating for this factor assumes that a recycling capability is provided for the glass process.

\subsection{Cost Effectiveness}

Six cost-related factors are identified explicitly for this criterion (Table 1). Three of these factors - lifecycle cost, investment cost, and utilization of existing infrastructure - are considered to be more important than the other three factors. 
Of the remaining three, establishment of product acceptability requirements, is more appropriately considered as a metric under investment cost. The factor "potential for cost sharing" is assigned zero weight because there is no valid information to support an assessment. The factor associated with cost uncertainty is folded into the investment and lifecycle cost factors. Finally, due to a lack of cost data, the investment cost and lifecycle costs are considered as one factor.

Bechtel and WSRC examined facility layout concepts for both the glass and ceramic processes as part of the ongoing EIS decision process (Ref. 25 - 28). However, at this point in these preconceptual design studies, there is insufficient detail to effectively distinguish between the investment or life cycle costs for the two baseline technologies; i.e., the costs for each production process are essentially the same within the uncertainty range of the existing engineering data. (A rough cost estimate for the conversion and first stage immobilization facility is $\$ 400-500$ million.) In the absence of discernible cost differences at the preconceptual design level, potential cost differences associated with certain common attributes of the two forms are identified and, in some cases, quantified.

\subsubsection{Investment and Life Cycle Cost: S - M (C) - Small to moderate cost} advantage for ceramic due primarily to the cost associated with the extra HLW canisters and the design and operational impacts associated with the higher radiation source term for glass.

Several potential areas of distinct cost differences were identified, including: (1) additional canisters of HLW forms for glass due to ceramic's higher density and plutonium loading, (2) facility design and operational impacts for factor-of-eight times higher radiation dose source for glass, (3) differences in waste form qualification and product control reflecting the glass experience with the DWPF "model," (4) potential higher development costs for the melter versus the MOXbased ceramic formation process, and (5) raw material (frit versus ceramic precursors) and equipment replacement costs. Life cycle cost differences in these areas are summarized for the two forms in Table 10.

Additional HLW Canisters for Glass. For the baseline and variant cases, the combination of differentials in the immobilization forms' densities and projected plutonium loadings results in substantial differences in the plutonium loading. This differential directly impacts the displacement of DWPF glass, independent of the canister loading strategy or total number of canisters involved, and is simply a measure of the additional DWPF canisters that must be poured as a result of the direct displacement of the DWPF HLW glass. Since the MD program will have to pay the repository a fee for each HLW canister placed in the repository, the difference in the number of additional canisters between glass and ceramic forms represents a real cost difference. 
On the basis of the baseline designs in terms of form size, plutonium loading, form density, and can dimensions, estimates were made of the additional canisters required for the baseline and enhanced plutonium loading cases for both glass and ceramic using the glass displacement curves presented in the LLNL TEP Presentation. For $10.5 \mathrm{wt} \%$ plutonium $\left(11.9 \mathrm{wt} \% \mathrm{PuO}_{2}\right)$ in ceramic and $8.8 \mathrm{wt} \%$ plutonium $\left(10 \mathrm{wt} \% \mathrm{PuO}_{2}\right)$ in glass, the differential is about 140 canisters. At the assumed program cost of $\$ 500,000$ per canister for repository disposal (Refs. 2, 4), this corresponds to an extra cost of about $\$ 70$ million for the glass immobilization form. This cost differential is a rough estimate based on the current baselines. Further optimization of form loading, can design, and canister loading and hardening concepts will likely change this cost differential. In addition, the cost per canister is currently under review by DOE/RW and is likely to increase.

Table 10. Areas of cost differences between glass and ceramic processes.

\begin{tabular}{|c|c|c|}
\hline Cost category & $\begin{array}{l}\text { Life cycle cost } \\
\text { difference }\end{array}$ & Advantage to: \\
\hline - Additional canisters & $\sim \$ 24 \mathrm{M}$ to $\$ 70 \mathrm{M}^{(1)}$ & Ceramic \\
\hline $\begin{array}{l}\text { - Higher Neutron Rate } \\
\text { - Enriched B-10 } \\
\text { - } \Delta \text { Automation } \\
\text { - } \Delta \text { Shielding and Space } \\
\text { for Maintenance } \\
\text { - ALARA }\end{array}$ & $\begin{array}{l}\sim \$ 17 \mathrm{M} \text { to } \$ 50 \mathrm{M}^{(1)} \\
\text { Not evaluated } \\
\text { Not evaluated } \\
\text { Not evaluated }\end{array}$ & $\begin{array}{l}\text { Ceramic } \\
\text { Ceramic } \\
\text { Ceramic } \\
\text { Ceramic }\end{array}$ \\
\hline - Form qualification & Not evaluated & Glass \\
\hline - Melter development & Not evaluated & $\begin{array}{l}\text { Ceramic; offset glass } \\
\text { advantage above }\end{array}$ \\
\hline - $\Delta$ Raw materials & $\sim \$ 6 \mathrm{M}$ to $\$ 18 \mathrm{M}^{(2)}$ & Ceramic \\
\hline - $\Delta$ Equipment replacement & Not evaluated ${ }^{(3)}$ & No discernible difference \\
\hline
\end{tabular}

(1) Range reflects the $17 \mathrm{MT}$ and $50 \mathrm{MT}$ feed cases

(2) Estimates reflect difference in the estimated glass frit and ceramics precursor costs.

${ }^{(3)}$ Costs for replacing the glass melter crucibles versus sintering furnace replacement.

Radiation Dose Impacts. Although not analyzed, cost differentials result from the considerably higher neutron dose rate associated with the glass form after the blending operation. As indicated in Section 5.5.1, the total radiation dose field is on the order of seven to eight times higher for the glass form and is dominated by neutron dose. This higher neutron dose rate, which is more difficult to shield than the soft gamma rays from plutonium, will impact facility design and operations, and in turn, result in higher costs in several areas. 
As discussed in Section 5.5, one approach for reducing the radiation dose for the glass form is to use boron enriched in the ${ }^{10} \mathrm{~B}$ isotope in the glass frit to reduce the alpha-neutron reaction. The enriched boron, estimated to cost about $\$ 3.50$ per gram, would result in a total cost increase for glass of $\$ 50$ million over the mission life for the 50 MT feed case.

DOE limits on personnel exposure, as discussed in Section 5.5, are more difficult to achieve for the glass process. Substantial automation is planned for the immobilization facility, and this will significantly aid in dose reduction. The bulk of the benefits gained occur for relatively simple automated operations that are cost effective. Because of the lower dose rate, there are potential opportunities in the ceramic process for some operated-assisted steps that could decrease equipment complexity and cost. If the high neutron dose rates from the immobilized glass form require additional automation, then higher costs are incurred. The total incremental cost for this additional automation includes both the added development and testing as well as the installation and operation of the enhanced system. Equipment concepts and layouts are not sufficiently advanced to determine the differences between the glass and ceramic process lines and their costs at this time.

Maintenance is a separate area where the higher neutron dose rates of glass can have an impact. Normally, SRS designs glovebox lines with an operating side and a maintenance side. The operating side is often well shielded. In the current plant concept, multiple processing stations (melters, for example) are placed in adjacent locations within a glovebox line. Routine maintenance procedures, not yet developed, may involve additional costs to ensure acceptable radiation exposure to maintenance personnel. Possible additional alternatives of increased station and glovebox spacing and additional shielding further add to the cost.

Immobilization Product Control and Qualification (Technical Maturity). The TEP review of technical maturity of the two forms (Ref. 11, Section 6.4) pointed out that LaBS glass belongs to the well-known family of borosilicate glasses qualified for HLW disposal and thus has an advantage over the ceramic form in the areas of product control and waste form qualification for the repository. If this advantage translates into significantly more development for the ceramic form, there could be both cost and timing impacts.

The argument for these advantages is two-fold and relies on the extensive experience at SRS with the HLW glass in the DWPF. First, a product control model has been developed based on an experimentally derived processing parameter envelope. A similar model could be applied to the LaBS glass form more readily than to the ceramic form. Second, the fact that the HLW glass has been qualified for repository disposal should make qualification of the LaBS plutonium-bearing glass easier and less expensive than qualification of the ceramic form. 
There are however, several counterpoints to this argument:

- The plutonium glass has a significantly different composition and formation parameters than the HLW glass. The baseline glass form is close to the plutonium concentration limit where some $\mathrm{PuO}_{2}$ precipitates from the LaBS glass under the thermal conditions experienced during the HLW glass pour into the canister (Ref. 11). This could affect requirements for product control and the glass form's qualification for the repository.

- The ceramic form can be easily sampled to ensure product quality, so extensive DWPF glass experience is not necessary.

- The waste form qualification requirements for repository disposal have not been established. If these are different from those for HLW glass, both forms will have similar pathways to acceptance. If they are similar, the ceramic form will benefit from the process established by glass.

The current R\&D plan allows sufficient time for both glass and ceramic form development and qualification. Whether there is a cost difference between the two is uncertain at this time.

Melter Development Costs. The current induction melter concept for the high temperature LaBS glass has not been developed and tested with plutonium feed materials. This complex piece of equipment requires more development than any of the ceramic system equipment components, which are in use in the MOX fuel industry with actinide materials. The resultant higher development costs of the melter, offset the advantage of the glass over ceramic in the areas of product control and qualification.

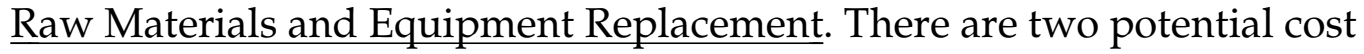
differentiators associated with raw materials and equipment replacement. First, the cost of the glass frit, estimated at $\sim \$ 31$ million ( $\$ 25 \mathrm{M}$ from Ref. 31 plus $\$ 6 \mathrm{M}$ for hafnium oxide) over ten years for the $50 \mathrm{MT}$ case, is greater than the cost of the $\mathrm{UO}_{2}$ and precursor oxides for the ceramic form $(\sim 13 \mathrm{M})$. Second, existing corrosion data for the platinum-rhodium crucible material suggest a maximum potential crucible lifetime of 1 to 1.3 years. For the $50 \mathrm{MT}$ case, this translates to a replacement requirement of about 45 to 70 melter crucibles during the production campaign. Replacement of the sintering furnaces or their components for the ceramic process will be less frequent and less costly. Estimates of the melter replacement costs have not been made.

\subsubsection{Utilization of Existing Infrastructure: I (C) - Insignificant advantage to} ceramic due to the lower dose potential. In an Environmental Impact Statement preconceptual design study by WSRC and Bechtel (Ref. 28 and 29), preliminary equipment layouts were developed for the 221-F Canyon building for the glass and ceramic baseline flowsheets. Either production system fits within the available space. However, the higher dose rates associated with the glass process 
might challenge the design of the glass glovebox system to meet the current dose requirements in this facility.

\subsection{Timeliness}

The goal of this criterion is to carryout disposition in a prompt and timely manner. Urgent action reduces risks of theft or diversion of weapons-usable material and sends a positive signal to Russia and to the international community regarding U.S. weapon dismantlement and material disposition. The factors under this criterion are: time to start disposition; time to complete disposition; and impacts on existing or future missions.

5.7.1 Time to Start Disposition: ND - No discernible difference between the two forms. The measurement used here to assess this factor is the date of, or time to, significant initiation of a "full scale demonstration." The current immobilization schedule calls for a "hot" demonstration at SRS in FY 2000 and actual production starting in the year 2005 (Figure 2). Both forms and processes are currently on a development track consistent with these startup dates. If sufficient funding is provided, the glass and ceramic technologies have a roughly equivalent likelihood of meeting the schedule.

There are two risk areas associated with immobilization technology maturity that cause uncertainty in the ability of the program to achieve this aggressive schedule:

- Waste form qualification - adequate time is provided in the schedule for either form to be qualified. On the basis of the previous discussion under cost impacts and the TEP report, there may be a lower risk for the glass form, depending on the specific waste form qualification requirements.

- Melter development - as indicated in Section 5.6.1, the melter represents the largest development challenge of any of the glass or ceramic process steps. It is currently believed that adequate time is provided in the R\&D schedule, but, there is a lower risk associated with comparable equipment development for the ceramic form.

These two risks are counter balancing for the two technologies.

5.7.2 Time to Complete Disposition: ND - No discernible difference. The metric for this factor is the time to process and immobilize the full inventory of surplus plutonium or, more specifically, the time to achieve the proliferation-resistant waste form, which would be stored pending repository disposal. Both forms and processes are currently under development consistent with a plant throughput of 5 MT per year. There appear to be no issues, other than those discussed under the previous section, with either form that result in a significant difference in their ability to meet the projected schedule. 
5.7.3 Impacts to Existing or Future Missions: I (C) - Insignificant advantage for ceramic. This factor measures the impacts on site and facility selection due to ongoing or already planned missions that affect the attractiveness of a site or facility. Should there be a potential conflict, the effect on relocating the plutonium immobilization mission or the conflicting mission must be taken into consideration. For comparing glass and ceramic forms, their relative impacts on the DWPF were considered.

Since the DWPF facility was assumed to be used to immobilize the plutonium glass-filled or ceramic-filled cans, there will be an impact on DWPF operations. The internal cans containing either form type will displace an equal volume of HLW glass in each canister. This, in turn, will reduce the quantity of high level waste (HLW) in each canister and, therefore, will increase the total number of canisters required to dispose of the SRS inventory of high level waste.

The nominal period for immobilization of $50 \mathrm{MT}$ of plutonium is 10 years (5 MT per year design throughput). The amount of plutonium in a plutonium-bearing canister (determined in part by the concentration of plutonium in the immobilized form) can affect the timeliness for completion of the immobilization mission in the following manner.

If the number of canisters with HLW glass generated per year to complete the plutonium immobilization mission in 10 years exceeds the rate per year at which DWPF can pour HLW glass, then the immobilization mission will be stretched out in time. The nominal DWPF glass pour rate planned for FY98 is limited to about 220 canisters per year by the amount of funding available to the program. This number of canisters per year is presently an upper bound on the number of canisters per year which the ProCast thermal code (Ref. 11) indicates can be successfully filled with HLW glass. Thus the plutonium form with the higher plutonium volumetric density (i.e., lower expected volume) will have a lower impact on the number of canisters required. As shown in Table 11, the volumetric plutonium density for the ceramic is considerably higher than for glass. It is important to note that there is an impact (and a discriminator between the glass and ceramic forms) only when the number of plutonium-bearing canisters exceeds the HLW disposal rate. At the current rate of 220 HLW canisters per year, this is not the case. However if either the HLW canister rate falls or the Pu disposition rate is raised, this could become an issue.

Table 11. Baseline plutonium volumetric loading.

\begin{tabular}{lcl}
\hline Cases & Ceramic & Glass \\
\hline Baseline \#1 (50 MT Pu/16 MT U) & $0.578 \mathrm{~g} / \mathrm{cm}^{3}$ & $0.334 \mathrm{~g} / \mathrm{cm}^{3}$ \\
Baseline \#2 (17 MT Pu/16 MT U) & $0.578 \mathrm{~g} / \mathrm{cm}^{3}$ & $0.334 \mathrm{~g} / \mathrm{cm}^{3}$
\end{tabular}




\subsection{Overall Assessment}

\subsection{Integrated Rating of Forms Against Criteria}

Table 12 presents a summary of the ratings made in Section 5.0 for the two candidate forms with respect to their advantages for specific factors under the decision criteria areas. Also shown in Table 12 are the criteria weights indicating the level of importance (from Table 1).

Table 12. Ceramic versus glass advantages for decision factors.

\section{Criterion - Factor}

1. Resistance to Theft or Diversion

a. Low Attractiveness

c. Minimize Processing

d. Safeguards and Security Assurance

e. Difficulty of Retrieval \& Extraction

2. Resistance to Reuse by Host Nation

a. Difficulty of Retrieval and Extraction

b. Assurance of Detection

3. Technical Viability
a. Technical Maturity
b. Viability Risk
c. Repository Acceptability of Disposal Form

4. Environmental, Safety, and Health Compliance
a. Public and Worker Health and Safety
b. \& d. Waste Minimization and Forms

5. Cost Effectiveness

a. \& b. Investment and Life Cycle Costs

e. Utilization of Existing Infrastructure

6. Timeliness
a. Time to Start Disposition
e. Utilization of Existing Infrastructure
c. Impacts to Existing or Future Missions

Weight (\%) Advantage*

22 Small $(C)$

Insignificant $(\mathrm{G})$

No Difference

Small (C)

Small to Med. (C)

19 Insignificant $(C)$

Insignificant (C)

No Difference

19 Small $(C)$

No Difference

No Difference

Small to Med.(C)

14 Small to Med. (C)

Small to Med. (C)

No Difference

11 Small to Med. (C)

Medium (C)

Insignificant $(\mathrm{C})$

$15 \quad$ No Difference

No Difference

No Difference

Insignificant $(\mathrm{C})$

* $\mathrm{G}=$ Glass; $\mathrm{C}=$ Ceramic 
Assessment of the information currently available indicates that both the ceramic and glass technologies provide acceptable forms for the immobilization of plutonium. As discussed in Section 5.0, the ceramic technology is superior to the glass technology because of the accumulation of small to moderate advantages for a number of important decision factors. These include proliferation resistance, repository performance and acceptability of waste form, potential worker dose, and cost effectiveness. Glass, on the other hand, has only a slight advantage for one nonproliferation factor (attractiveness level) as well as some small advantages for specific metrics that are offset by ceramic advantages for competing metrics within the same factors.

On the basis of the results of both the TEP evaluation and this overall assessment, the ceramic form on the whole is preferred over the glass form. As discussed below, this recommendation of the ceramic technology for plutonium immobilization, on the basis of its superior technical merit, is made with reasonable confidence that this form and process can be successfully implemented.

\subsection{Robustness of Recommendation and Sensitivity Analysis}

The confidence level of LLNL's recommendation of the ceramic technology is assessed below on the basis of: (1) the relative robustness of the ceramic technology against technical and programmatic uncertainties, and (2) the sensitivity of the ceramic-over-glass combined score to uncertainties in the weights and ratings.

\subsubsection{Robustness to Programmatic and Technical Uncertainties}

As concluded by the TEP and this evaluation, both the ceramic and glass technologies have sufficiently high levels of maturity to provide a reasonably high level of confidence in the successful implementation of either form. However, there are several programmatic and technical uncertainties that could affect the implementation of the chosen form. For these, the ceramic technology is either more robust or comparable to glass.

HLW Glass Pouring Restriction Due to the Internal (Immobilization Form) Cans and Rack Assembly. The current can-in-canister configurations for the ceramic and glass forms were chosen based on ProCast model analyses of thermal and fluid flow properties of the HLW glass. Because the code has not been validated by large-scale glass pour tests, there is uncertainty in the number of cans of plutonium form that can be accommodated within the canister. Furthermore, as discussed in Section 5.7.3, there may not be a sufficient number of HLW canisters available each year to meet the Immobilization Program's objective schedule, especially if a lower volume occupancy fraction for the forms is required. This could occur if the canister configuration is structurally enhanced 
to thwart theft and diversion. Because of the ceramic form's significantly higher plutonium density (Table 11), the ceramic form has a much greater margin for accommodating these uncertainties.

Unknown Waste Form Acceptance Requirements. As discussed in Section 5.4, the ceramic form may have a slight advantage due to its greater durability. On the other hand, if the requirements and qualification process are similar to those for DWPF glass, then the glass form would have a small advantage due to the existing model of relating process and product control to acceptance requirements for product.

Uncertainty in Range of Feed Impurity Levels. Both forms have demonstrated the ability to accommodate impurities at levels that exceed the expected range. However, both forms will require additional formulation testing to define the feed compositional and processing parameter envelopes that will guarantee an acceptable product. In this regard, the glass form has a small advantage, but the ceramic form has no identifiable issues that affect its timely development.

Possible Requirements for Enhanced Intrinsic Proliferation Resistance of the Canin-Canister Configuration. Both the ceramic and glass forms have flexibility to accommodate the diversion-resistant concepts discussed in Section 4.4. Because of its volumetric advantage, the ceramic form more readily allows for such structural enhancements as armoring.

Additional Plutonium Materials ( $>50 \mathrm{MT}$ ) for Disposition. Both technologies could accommodate plutonium feed in excess of the 50-MT case by extending the operating campaign of the immobilization plant beyond ten years. By virtue of its greater ability to increase the plutonium concentration of the form and its higher density (x1.7), the ceramic form has greater flexibility for increasing the plutonium throughput in the immobilization plant, thereby reducing the length of an extended production campaign.

\subsubsection{Sensitivity Analysis}

Sensitivities to the ceramic-over-glass result were evaluated by using simple qualitative logic and by performing a "quantitative" sensitivity analysis.

On a qualitative basis, making the glass form technically superior to ceramic requires eliminating a majority of the favorable ceramic ratings noted in Table 12 and introducing three or four ratings favorable to glass. This is not realistic, considering the following factors:

- Worker exposure: the factor-of-eight difference in radiation field favors ceramic for reason of ALARA if all other factors were equal. A new glass form would have to be developed without boron, a major constituent in the LaBS glass, to levelize the ratings for this factor. This would introduce an 
unacceptable delay of one to two years (LaBS glass has been under development for about two years).

- Form durability: definite advantage to ceramic for long term repository performance. The titanate-based ceramic form is inherently more stable, even after radiation damage saturation, than the silicate-based glass forms.

- Cost differentials: - cost differentials associated with the larger number of canisters and design and operational provisions to mitigate glass's higher neutron dose are significant ( i.e., $>\$ 100$ million). On the basis of current knowledge of the respective flowsheets and equipment for the two technologies, this cost advantage is not expected to change in favor of glass.

Because the ceramic technology rates equal to or higher than glass for all but one of the decision factors, a quantitative sensitivity analysis would not be particularly revealing for the set of criteria considered here. However, it is useful to assess the sensitivity of the overall technical rating of the two technologies to potential institutional factors which were not considered in this assessment, such as public and regulatory acceptance of a new waste form and acceptance by the Russian government of the proposed immobilized form. Such a sensitivity analysis was performed and the results are summarized in Appendix A. The sensitivity analysis indicates that the weight assigned to the institutional factor and the assumed advantage assigned to glass would have to be quite large to outweigh the technical advantages of the ceramic technology. For example, glass would have to be assigned a medium advantage and more than one-third of the total weight given to the institutional factor for the glass form to have a higher overall score.

\subsection{Peer Review Comments}

The external Peer Review Panel (PRP) met at LLNL August 18-21, 1997 to review this recommendation report, supporting documentation, and technical data. The panel reviewed the (second) partial draft of the TEP report and the initial draft of this recommendation report. The panel also considered additional documentation and presentation material from the TEP review, including presentations by the advocates for both the glass and ceramic immobilization technologies. The findings, conclusions, and recommendations of the PRP are contained in a letter report, dated August 21, 1997 (Ref. 12).

In general, the PRP agreed with the findings of this assessment and the TEP evaluation (Ref. 11), and endorsed the LLNL recommendation that DOE should implement the ceramic immobilization technology due to its superiority over glass in several key performance areas. The panel pointed out that the data for the evaluations of the two technologies were limited, but still sufficient to support the recommendation of the ceramic form. For the most part, the panel's independent assessment of the two technologies with respect to the decision criteria and factors verified the LLNL assessment and ratings for the two forms 
documented in this report. There were some minor differences for some factors regarding whether an advantage for the ceramic form was small or moderate, but overall, the PRP's qualitative ratings of advantages tracked those discussed in Section 5.0.

The PRP made a few recommendations in the letter report and provided several informal suggestions to LLNL program management during the course of the peer review that helped clarify information in the recommendation report. Many of these informal comments are reflected in this version of the report. One of the panel's formal recommendations is discussed below.

The PRP suggested that, because of the "early stage of development of both forms and their associated processes," proceeding with one form at this time represents "some risk that unexpected difficulties" could impact cost and schedule. On this basis, the panel recommended that a modest level of support be continued for the glass technology.

The immobilization program management at LLNL does not agree with this recommendation for three reasons. First, current guidance from DOE/MD indicates that the immobilization budgets for FY 1998 and FY 1999 will be at levels that can only support the continued development of one immobilization technology. Unless these budget levels are increased by a few million dollars, performing any useful level of development of the glass technology as a backup would result in a schedule slippage of the project by at least one year. Second, LLNL believes that the cost and schedule risks are sufficiently low for the ceramic technology that any possible delays or increased costs associated with "unexpected difficulties" in implementing the ceramic technology would be lower than the added costs and time required to implement the glass technology (assuming it receives modest development as a backup) once such difficulties were encountered. Finally, LLNL and the panel agree that there are no "showstoppers" in implementing the ceramic technology. Thus, the likelihood of needing the glass technology is extremely small.

\subsection{Conclusion}

On the basis of comprehensive technical evaluations of the ceramic and glass immobilization technologies, LLNL recommends that the ceramic technology be developed for deployment in the planned plutonium immobilization plant. Both candidate technologies are acceptable for the plutonium immobilization mission, but the ceramic technology offers a number of important advantages over glass, notably:

- The ceramic form is somewhat more robust to the threat of theft, diversion, or reuse because the extraction of plutonium from the final form is more complicated and neither fully developed nor published. 
- The ceramic form is expected to be more durable in a repository environment and should retain plutonium and its decay products for longer periods of time.

- The ceramic form has a significantly lower radiation source term that reduces the potential for worker exposure during fabrication before insertion into high-level waste canisters.

- The ceramic form and process offers potentially significant cost savings relative to the glass technology.

- The ceramic technology is more flexible and can better accommodate modifications to programmatic and technical requirements.

The maturity of the ceramic technology, is comparable to that of glass and is sufficient to provide DOE with a reasonably high level of confidence that plutonium immobilization can be carried out successfully on the desired schedule. There are no "show stopper" issues for the ceramic technology (or for the glass form).

The results of the TEP, LLNL management, and PRP evaluations are generally consistent. The PRP findings validate the results of the TEP assessment and those presented in this report and endorse the LLNL recommendation for the deployment of the ceramic technology. 


\section{References}

1. Management and Disposition of Excess Weapons Plutonium, National Academy of Sciences, National Academic Press, Washington, D.C., 275 pp. (1994).

2. Record of Decision for the Storage and Disposition of Weapons-Usable Fissile Materials Final Programmatic Environmental Impact Statement, U.S. Department of Energy, January 14, 1997.

3. Alternative Technical Summary Report: Vitrification Greenfield Variant, L-202151, UCRL-122663, August 26,1996.

4. Alternative Technical Summary Report: Vitrification Can-in-Canister Variant, L-20216-1, UCRL-122659, August 26,1996.

5. Alternative Technical Summary Report: Vitrification Adjunct Melter to DWPF Variant, L-20217-1, UCRL-122660, August 26,1996.

6. Alternative Technical Summary Report: Ceramic Greenfield Variant, L-20218-1, UCRL-122662, August 26,1996.

7. Alternative Technical Summary Report: Ceramic Can-in-Canister Variant, L-20219-1, UCRL-122661, August 26,1996.

8. Alternative Technical Summary Report: Electrometallurgical Treatment Variant, L-20220-1, UCRL-122664, August 26,1996.

9. Technical Summary Report for Surplus Weapons-Usable Plutonium Disposition, Office of Fissile Materials Disposition, U.S. Department of Energy, DOE/MD0003, October 31,1996, Rev. 1.

10. Gray, L. and Gould, T., "Immobilization Technology Down-Selection Radiation Barrier Approach," Lawrence Livermore National Laboratory, UCRL-ID-127320, May 23, 1997.

11. Technical Evaluation Panel Summary Report: Ceramic and Glass Immobilization Options, DRAFT Rev. 1, Lawrence Livermore National Laboratory, August 8, 1997.

12. Peer Review Panel Report on the Down-Selection of Glass and Ceramic Pu Immobilization Forms, Lawrence Livermore National Laboratory, August 29, 1997.

13. Nonproliferation and Arms Control Assessment of Weapons-Usable Fissile Material Storage and Plutonium Disposition Alternatives - Department of Energy Office of Arms Control and Nonproliferation, DOE/NN-0007, January 1997.

14. The Office of Fissile Materials Disposition FY97 Annual Operating Plan, Rev. 4, U. S. Department of Energy, April 1997. 
15. Integrated Immobilization Plan for Research, Development, and Testing, DRAFT, Lawrence Livermore National Laboratory, April 1997.

16. Summary Report of the Screening Process - To Determine Reasonable Alternatives for Long-Term Storage and Disposition of Weapons-Usable Fissile Materials, U. S. Department of Energy Office of Fissile Materials Disposition, March 29, 1995.

17. Cygelman, A., U. S. Department of Energy, Letter to T. Gould on Criteria Weights, August 12, 1997.

18. Department of Energy Notice of Intent: Surplus Plutonium Disposition Environmental Impact Statement, [6450-01-P], U. S. Department of Energy, May 16, 1997.

19. Feed Materials Planning Basis for Surplus Weapons-Usable Plutonium Disposition - Predecisional Draft, U. S. Department of Energy, Office of Fissile Materials Disposition, April 2, 1997.

20. The Evaluation and Selection of Candidate High-Level Waste Forms, Savannah River Operations Office, August 1981 (Published: March 1982).

21. Gray, L., et. al., "An Analysis of Probable Clandestine and Host Country Plutonium Recovery Methods from Immobilized Forms,” Work in Progress.

22. Proliferation Vulnerability Red Team Report Draft, Sandia National Laboratories, August 1996.

23. Degraded Mode Criticality Analysis of Immobilized Plutonium Waste Forms in a Geologic Repository, TRW, AOOOOOOOO-01717-5705-00014, Rev. 01, February 7, 1997.

24. Civilian Radioactive Waste Management System, Total System Performance Assessment, 1995: An Evaluation of the Potential Yucca Mountain Repository, DI: BOOOOOOO-1717-2200-000136, Rev. 01, 1995.

25. EIS Data Call Input Report: Plutonium Immobilization Plant Using Ceramic in New Facilities at SRS, Lawrence Livermore National Laboratory, August 7, 1997.

26. EIS Data Call Input Report: Plutonium Immobilization Plant Using Glass in New Facilities at SRS, Lawrence Livermore National Laboratory, August 12, 1997.

27. EIS Data Call Input Report: Plutonium Immobilization Plant Using Ceramic in Existing Facilities at SRS, Lawrence Livermore National Laboratory, August 13, 1997.

28. EIS Data Call Input Report: Plutonium Immobilization Plant Using Glass in Existing Facilities at SRS, Lawrence Livermore National Laboratory, August 7, 1997. 
29. Lee, J. D., "Neutron Source \& Neutron Dose Estimates for Glass \& Ceramic Pu Immobilization Forms," Presentation to the TEP, Lawrence Livermore National Laboratory, Livermore, CA, July 28, 1997.

30. Rainisch, R., Westinghouse Savannah River Company, "Radiological Analysis of Plutonium Bearing Batches with High Americium Content," Inter-office Memorandum to E. N. Moore, April 3, 1997.

31. McKibben, M., Immobilization Program Peer Review, Lawrence Livermore National Laboratory, April 24-25, 1996 (Viewgraphs from presentation). 


\section{Appendix A Sensitivity Analysis}

The decision factor qualitative scores shown in Table 12 and factor weights assigned in Table A.1 were used to perform a sensitivity analysis of the overall rating of the glass and ceramic technologies. For the purposes of this sensitivity analysis, the qualitative scores ND, I, S, M, and L assigned in Section 5.0 are represented numerically as $0,0,0.25,0.5$, and 0.75 differences in scores on a 0 to 1.0 scale. For example, a qualitative score of "ND" translated into a score of 1.0 for both the ceramic and immobilization forms, and a qualitative score of " $\mathrm{S}$ " favoring the ceramic form translated into a score of 1.0 for the ceramic and 0.75 for the glass form. The overall figure of merit for the assessment is a weighted sum of the scores of the alternative for the factors. The weighted scores are shown in Figure A1.

Figure A1. Weighted scores of immobilization alternatives.

$\begin{array}{ll}\text { Alternative } & \text { Utility } \\ \text { Ceramic } & 1.000 \\ \text { Glass } & 0.785\end{array}$

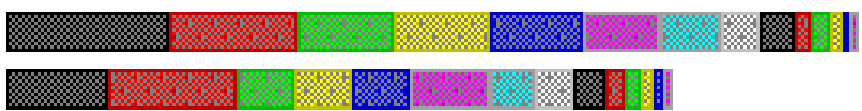

Repository accept
Radiation exposure
娄 Safeguards
Attractiveness
Waste min.

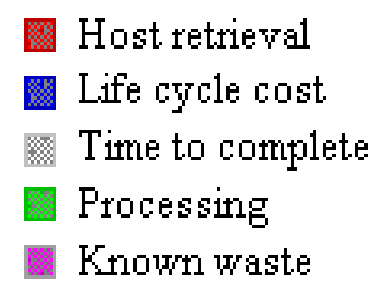

Terrorist retrieval

Time to start

Detectability-host

Mission impacts

Other

Figure A1 displays a stacked bar graph for each of the two immobilization forms. The overall score, labeled "Utility" is a weighted sum of scores for the factors shown in Table 12. The stacked bars shown in the figure are segmented to show the relative contributions of these scores for each alternative. For example, the first segment for the ceramic form is wider than the corresponding segment for the glass form. This indicates that the ceramic form scores better than the glass form on the "Repository acceptability" factor. The width of this segment reflects both the score of the alternative on the factor and the weight assigned to that factor.

The recommendation of the ceramic form over the glass form is extremely robust with respect to the weights assigned to the factors. Because the ceramic numerical scores are equal to or better than the glass numerical scores on all 
factors, no combination of weights assigned to the factors shown in Table 12 would lead to a rank reversal. The ceramic form is preferred under all possible sets of weights. Thus, a sensitivity analysis with respect to the weights assigned to the technical factors is not particularly revealing.

However, it is useful to assess the sensitivity of this technical recommendation to institutional factors, which were not explicitly considered in this assessment (per DOE/MD's guidance, Ref. 17). The TEP report did suggest that licensing agencies and other stakeholders are more familiar with glass immobilization forms and thus, might be more supportive of glass. This familiarity might facilitate licensing and acceptance of immobilization facilities and the mission. To explore the potential impact of such institutional acceptance, an additional factor was introduced into the model and a score of " $\mathrm{M}$ " favoring the glass form was assumed (i.e., glass $=1.0$, ceramic $=0.5$ ). The weight assigned to the institutional acceptance factor was then increased to identify the point at which institutional considerations would outweigh technical factors with the result that the glass form would be preferred over the ceramic form. Results are shown below in Figure A2.

\section{Figure A2. Sensitivity to weight assigned to added Institutional Acceptance factor .}

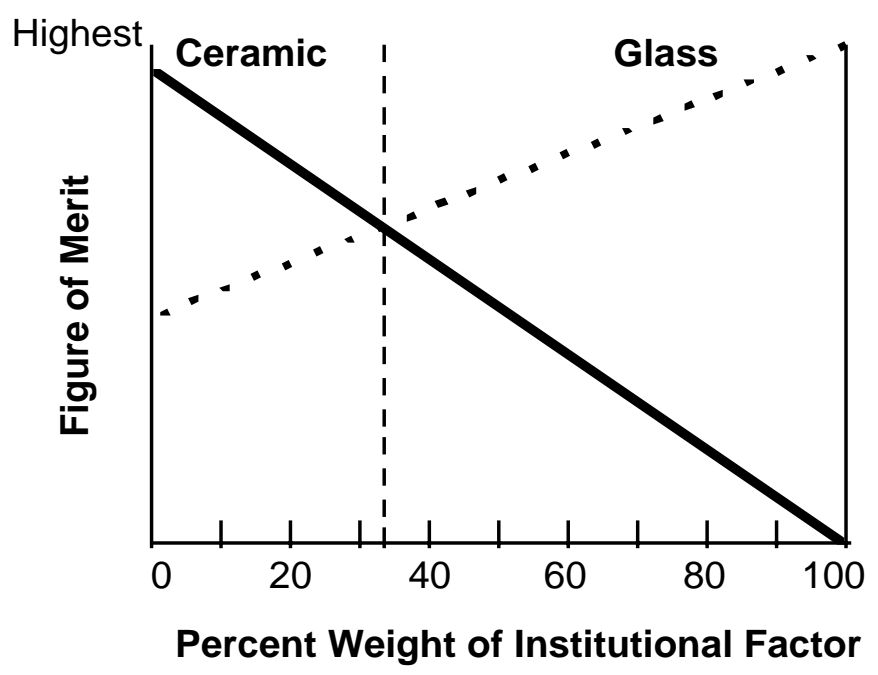

Figure A2 shows that the weight assigned to the "Institutional Acceptance" factor would have to increase to over $30 \%$ in order to make glass the preferred form. This would be the highest-weighted factor in the analysis. Thus, the ceramic preference is also robust with respect to institutional considerations. 
Table A1. Technology scores and weights for decision factors.

\section{Criterion - Factor}

1. Resistance to Theft or Diversion
a. Low Attractiveness
c. Minimize Processing
d. Safeguards and Security Assurance
e. Difficulty of Retrieval \& Extraction

2. Resistance to Retrieval, Extraction, and Reuse by the Host Nation
a. Difficulty of Retrieval, Extraction, and Reuse
b. Assurance of Detection

$\begin{array}{ll}1.0 & 1.0 \\ 1.0 & 1.0\end{array}$

3. Technical Viability
a. Technical Maturity
b. Viability Risk
c. Repository Acceptability of Disposal Form

4. Environmental, Safety, and Health Compliance

a. Public and Worker Health and Safety

$\begin{array}{ll}1.0 & 1.0 \\ 1.0 & 1.0\end{array}$

$1.0 \quad 1.0$

$1.0 \quad 0.625$

$1.0 \quad 0.625$

$\begin{array}{cc}\begin{array}{c}\text { Ceramic } \\ \text { score }\end{array} & \begin{array}{c}\text { Glass } \\ \text { score }\end{array} \\ & \\ & \\ 1.0 & 1.0 \\ 1.0 & 1.0 \\ 1.0 & 0.75 \\ & \\ 1.0 & 0.625\end{array}$

Weight

(\%)

(22) Reference 17

2 Inherently small differences between forms

2 Processing within secure, safeguarded facility

7 Important to assure no diversion \& facilitate IAEA inspections

11 Important for meeting Spent Fuel Standard and International Community perception

\section{Reference 17}

15 Important for meeting spent fuel standard and international community perception

4 Detection of large-scale recovery operations highly likely

(19) Reference 17

0 Evaluated in terms of cost/schedule impacts

0 Evaluated in terms of cost/schedule impacts

19 Receives entire weight of criterion per Ref. 17

(14) Reference 17 
b. \& d. Waste Minimization and Forms

5. Cost Effectiveness

a. \& b. Investment and Life Cycle Costs

e. Utilization of Existing Infrastructure

6. Timeliness

a. Time to Start Disposition

e. Time to Complete

c. Impacts to Existing or Future Missions
1.0

0.5

$1.0 \quad 1.0$

1.0

1.0

1.0

1.0
3 Indirect impacts to health and safety of people

(11) Combined high relative weight for two metrics of interest 10

1 Cost impacts under $a$ and $b$

\section{(15) Reference 17}

9 Initiation of disposition paramount

4.5 Total duration of lower importance

1.5 Secondary effect on timeliness 


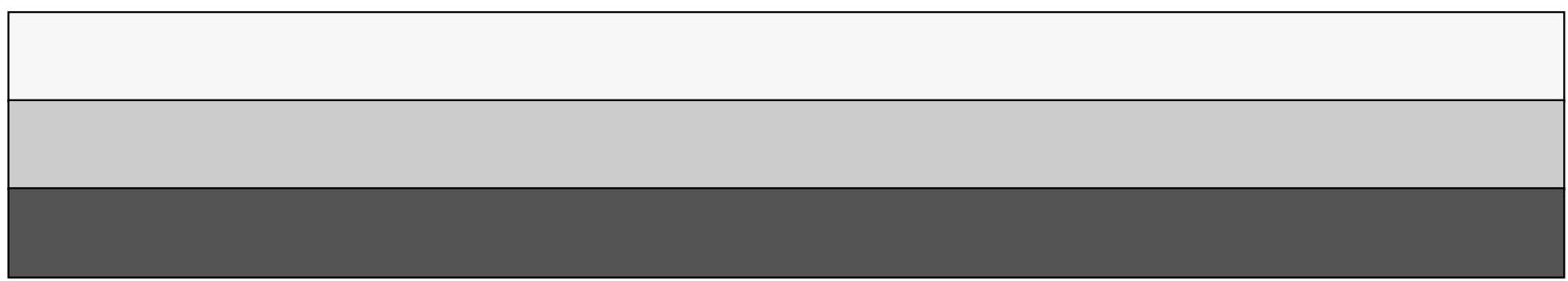

